\title{
Consensus Control Design for 360 MN Extrusion Machine Producing Process
}

\author{
Chao Wang, ${ }^{1}$ Wanzhou Li, ${ }^{1}$ Qianchuan Zhao, ${ }^{1}$ Fei Peng, ${ }^{1}$ Hongbin Yang, ${ }^{1}$ and Rui Huang ${ }^{2}$ \\ ${ }^{1}$ Department of Automation and Tsinghua National, Laboratory for Information Science and Technology (TNList), \\ Tsinghua University, Beijing 100084, China \\ ${ }^{2}$ Fitter Weld Pilot Pipe Plant, Chengdu Aircraft Industrial (Group) Company Limited, Chengdu 610092, China
}

Correspondence should be addressed to Qianchuan Zhao; zhaoqc@tsinghua.edu.cn

Received 27 November 2013; Accepted 23 January 2014; Published 13 March 2014

Academic Editor: Fuzhong Nian

Copyright (C) 2014 Chao Wang et al. This is an open access article distributed under the Creative Commons Attribution License, which permits unrestricted use, distribution, and reproduction in any medium, provided the original work is properly cited.

\begin{abstract}
This paper mainly addresses the issue of $360 \mathrm{MN}$ extrusion machine and focuses on the stabilization control of main table attitude. We will first introduce the problem and then model the extrusion machine. As the machine is a multi-input multioutput (MIMO) and strong coupling system, it is challenging to apply existing control theory to design a controller to stabilize the main table attitude. Motivated by recent research in the field of multiagent systems, we design a consensus control protocol for our system and derive our convergence conditions based directly on Routh stability criterion. The advantages of the design are also demonstrated by numerical simulation.
\end{abstract}

\section{Introduction}

Thick-walled seamless tubes are extremely important to the development of some heavy industries. With higher performance of heat-resisting and pressure-resisting, the thickwalled seamless tubes are widely used to transfer liquid in the fields of ships, chemicals, power plants, petroleum, and other industries. 36,000-ton (360 MN) extrusion machine is the world's biggest ferrous metal vertical extrusion press machine, as shown in Figure 1, which has been put into production in Baotou, China, in 2009. In Figure 1, the left side figure shows process of squeezing tube billet by driving activity crossbeam upward, the upper right figure is high temperature tube billet formed by extrusion, and the low right figure is finished product. The $360 \mathrm{MN}$ extrusion machine can make large-diameter thick-walled seamless tubes for over $600 \mathrm{MW}$ ultrasupercritical and nuclear power plants. Extrusion is a popular mode for steel production. Through the mold fixed on pressure machine, extrusion table pressures the metal blank into the mold cavity to deform the plastic and to obtain the required size, appearance shape, and certain mechanical properties. During the extrusion, the movement and modeling of the metal blank are controlled by the mold.
The $360 \mathrm{MN}$ extrusion machine uses one-step forming technology to make thick-walled tubes. The materials include high strength alloy steel, stainless steel, refractory alloys, and special metals like uranium, titanium, and niobium. The $360 \mathrm{MN}$ extrusion machine improves the production efficiency greatly. A single extrusion process takes $40 \sim 60 \mathrm{sec}-$ onds. The $360 \mathrm{MN}$ extrusion machine is typical hydromechatronics integration equipment. The main table is pushed upward by six main cylinders underneath, which extrude out the tube. To generate qualified products, we have to design a control strategy to generate the main table's attitude in horizontal direction.

Researches on the relevant fields of extrusion attract more and more attention in recent years. Tibbetts and Wen put forward that the complex material extrusion process can be viewed as three stages including Material Response, Thermomechanical Response, and Press Response in [1]. Moreover, they developed a model of the extrusion process specifically for real-time control and figured out the optimization of extrusion control. Researchers have analyzed means to measure the internal temperature in the cross section of the polymer extrusion which is critical to stabilization process [2]. However, due to high-power, long-loop, complex 


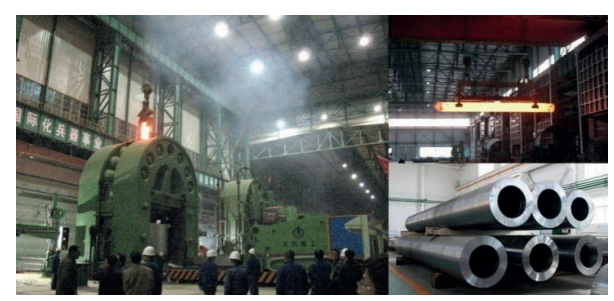

Figure 1: The $360 \mathrm{MN}$ vertical extrusion press and the largediameter thick-walled seamless tubes.

extrusion process characteristics, some new problems come up to $360 \mathrm{MN}$ extrusion machine compared with the ordinary ones.

In this paper, we try to solve the control design problem through a simplified model in which the six main cylinders are regarded as six strong coupling agents. We then introduce consensus control method to keep the balance of main table. Development of consensus control can be traced back to 1960s [3]. As time goes on, researchers began to use graph Laplacians and their relevant properties to analyze converge of consensus and agents' position or velocity. Based on the theory of Graph Laplacians, Olfati-Saber et al. provide a theoretical framework to analyze consensus algorithms for multiagent networked systems [4]. Recently, researchers pay close attention to branches of consensus control, such as behavior of flocks and swarms [5], nonlinear mathematical models [6], optimization of consensus algorithm [7], the effect of the fuzzy sliding-mode [8], and the communication time-delay effect [9]. Moreover, the situation where every agent is a multidimensional dynamic system has been considered by Wen and Chen [10]. On the other hand, researchers keep a closer eye on experimental studies. Ren and Chao and Bourgeous proposed a mobile actuator and sensor network platform, which can validate the correctness of Consensus Algorithms for Multivehicle Cooperative Control under different conditions [11]. We also utilized a mechanismhydraulic cosimulation platform constituted by Adams and Easy5 software [12], which establishes a simulation platform for the design and evaluation of the control system of a $360 \mathrm{MN}$ extrusion machine. The simulation results of the cosimulation platform capture the device characteristics of mechanical and hydraulic systems.

This paper is organized into seven sections. Section 2 describes the system of the huge extrusion machine, builds a mathematic model, and states the problem when applying the existing control strategy. Section 3 designs a new control strategy based on the consensus control. In Section 4, we establish the convergence condition of the new consensus control strategy. Then, in Section 5, several simulations have been conducted on the mathematic platform to examine the result of the theoretical derivation. In Section 6, more simulations are provided on a proved cosimulation platform which is believed to be closer to the reality. Finally, the conclusion is summarized in Section 7.

\section{Problem Statement}

2.1. Problem Description. Figure 2 shows the hydraulic system layout. The main pumps are placed on the B1 floor $(-7$ meters); the tanks and auxiliary pumps are placed on the B2 floor (-14 meters). Forty-two WEPUKO RX500 Radial Piston Variable pumps drive the extrusion process with maximum working pressure $420 \mathrm{bar}$. A pump provides $1000 \mathrm{~L} / \mathrm{min}$ flow at 350 bar or $750 \mathrm{~L} / \mathrm{min}$ at 420 bar. The movement of the pump's embedded piston causes the oscillation of the cam, making the desired change of radial piston position and, as a result, the desired change of the flow. The piston movement is controlled by a MOOG servo valve. The forty-two pumps are divided into six groups, each one of which is assigned to one $1350 \mathrm{~mm}$ diameter main cylinder.

The hydraulic control system of $360 \mathrm{MN}$ extrusion machine is shown in Figure 3. Four SSI digital position transducers placed at the four corners collect the main table position data and transfer the signal to the central controller. Meanwhile, six pressure transducers in each main cylinder collect the extrusion force signals and transfer it to the controller as well.

The control system output is the set point value for the servo valve PID controller AN430, which, together with the servo valve, piston, and variable pump, comprises a typical electrohydraulic position control system.

The major disturbance in this control system is the uncertain resistance force during extrusion process, due to temperature asymmetry of the ingot, metal turbulent, inconformity among cylinders, and many others. These disturbances will result in the unbalance of the main table, which will lead to the decline of the products. The control purpose is to ensure the horizontal balance of the main table which is actuated upward by a set of main hydraulic cylinders symmetrically installed around the circular plane. In practice, open loop control is used. The disadvantage of open loop control is obvious: performance will become poor when the system faces unexpected nonlinear resistance.

2.2. Mathematical Model of the Machine. In order to study the control of the $360 \mathrm{MN}$ machine, we introduce a simplified mathematical model of the system. The actual dynamics of the model is much more complicated. We built a more realistic simulation model in Adams and Easy5 to test the control design. The details of the simulation model can be found [12].

Abstract the main table of the extrusion machine into a circular plane. Assume that $N$ points are uniformly distributed around the circular plane. At each point $i$, there is an upward force driven upward. The center of the circle is fixed on the horizontal plane. Define the axis and axis on the circular plane as below. The number 0 point locates on the negative direction of axis $\alpha$. Because the $N$ points are uniformly distributed, assume that the angular interval is $\delta=2 \pi / N$, so the angular between the vector, composed of the center of circle to the $i$ point, and the negative direction

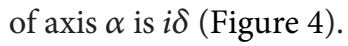




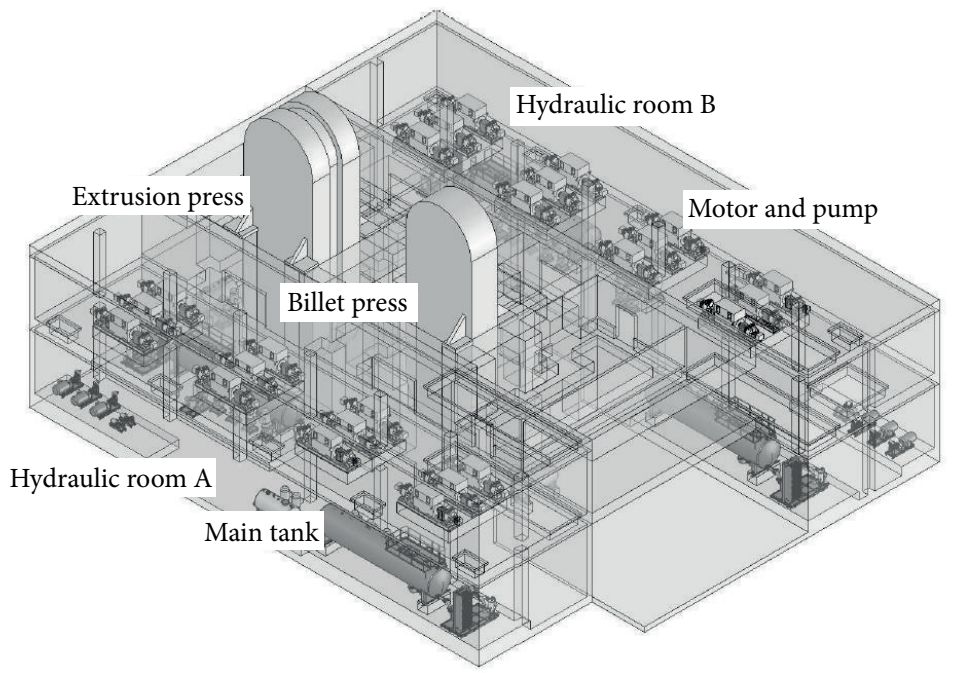

FIGURE 2: The hydraulic system layout.

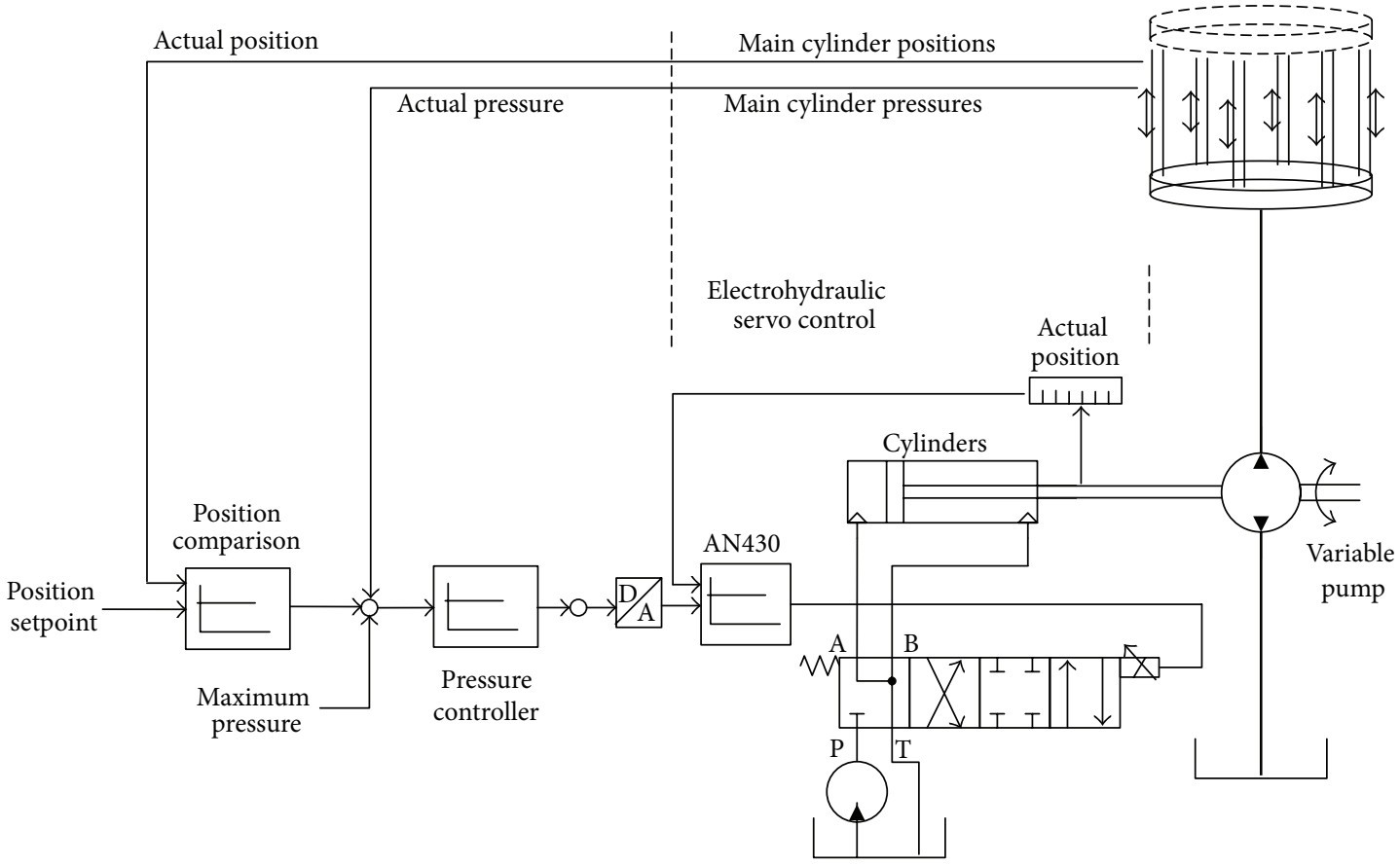

FIgURE 3: The hydraulic control system.

Suppose the radius of the circle is $r$, the angular deviation of axis $\alpha$ is $\varphi_{\alpha}$, and the angular speed of axis $\alpha$ is $\omega_{\alpha}$. The angular deviation of axis $\beta$ is $\varphi_{\beta}$, and the angular speed of axis $\beta$ is $\omega_{\beta}$. Let the total gravity of the main table be $G=m g$ and assume it is equally shared by the $N$ cylinders (Figure 5). Then the torque of the force $F_{i \delta}$ and the shared gravity $G / N$ by cylinder $i$ projected at the axis $\alpha$ is

$$
M_{i \alpha}=\left(F_{i}-\frac{G}{N}\right) \cdot r \cdot \sin (i \delta)
$$

So we can get the torque of all force projects at the axis $\alpha$ as

$$
M_{\alpha}=\sum_{i=1}^{N} M_{i \alpha}
$$

From the angular momentum, we can get

$$
M_{\alpha} \cdot d t=I_{\alpha} \cdot d \omega_{\alpha}
$$




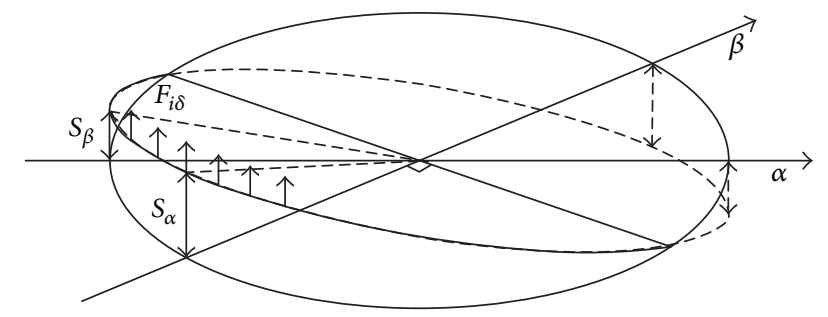

Figure 4: The space structure of the circular plane mathematic model.

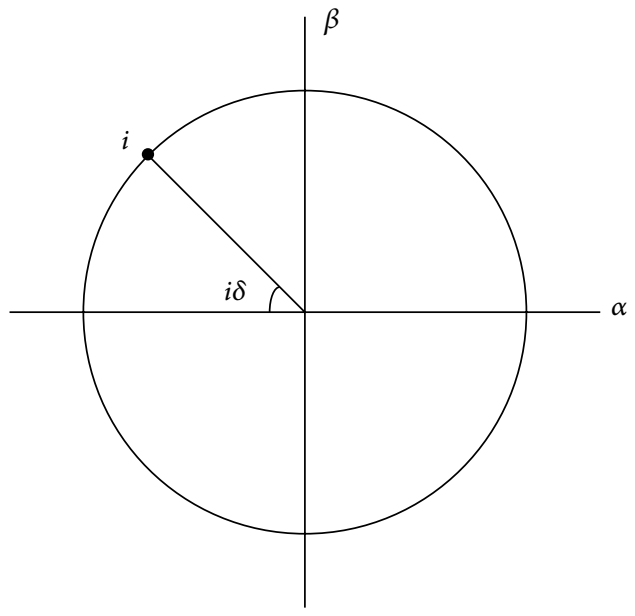

Figure 5: The projection on the horizontal plane of the plane mathematic model.

where $I_{\alpha}=(1 / 2) m r^{2}$; set $m=1$, so

$$
\frac{d \omega_{\alpha}}{d t}=\frac{M_{\alpha}}{I_{\alpha}}
$$

Similarly, the torque of the force $F_{i}$ project at the axis $\beta$ is

$$
M_{i \beta}=F_{i} \cdot r \cdot \cos (i \delta) .
$$

The total torque for axis $\beta$ is

$$
M_{\beta}=\sum_{i=1}^{N} M_{i \beta} .
$$

From the angular momentum, we can get

$$
M_{\beta} \cdot d t=I_{\beta} \cdot d \omega_{\beta},
$$

where $I_{\beta}=(1 / 2) m r^{2}$; set $m=1$, so

$$
\frac{d \omega_{\beta}}{d t}=\frac{M_{\beta}}{I_{\beta}} .
$$

We also have

$$
\begin{aligned}
& \frac{d \varphi_{\alpha}}{d t}=w_{\alpha}, \\
& \frac{d \varphi_{\beta}}{d t}=w_{\beta} .
\end{aligned}
$$

So, the dynamics of the plane under input force at the cylinders can be summarized as

$$
\begin{gathered}
\dot{\omega}_{\alpha}=\frac{\sum_{i=1}^{N}\left(F_{i}-G / N\right) \cdot r \cdot \sin i \delta}{I_{\alpha}}, \\
\dot{\omega}_{\beta}=\frac{\sum_{i=1}^{N}\left(F_{i}-G / N\right) \cdot r \cdot \cos i \delta}{I_{\beta}}, \\
\dot{\varphi}_{\alpha}=\omega_{\alpha}, \\
\dot{\varphi}_{\beta}=\omega_{\beta},
\end{gathered}
$$

which is actually the state equation of the system. In matrix form, we have

$$
\left[\begin{array}{c}
\dot{\varphi}_{\alpha} \\
\dot{\omega}_{\alpha} \\
\dot{\varphi}_{\beta} \\
\dot{\omega}_{\beta}
\end{array}\right]=A\left[\begin{array}{c}
\varphi_{\alpha} \\
\omega_{\alpha} \\
\varphi_{\beta} \\
\omega_{\beta}
\end{array}\right]+B\left[\begin{array}{c}
F_{1}-\frac{G}{N} \\
\vdots \\
F_{N}-\frac{G}{N}
\end{array}\right],
$$

where

$$
A=\left[\begin{array}{llll}
0 & 1 & 0 & 0 \\
0 & 0 & 0 & 0 \\
0 & 0 & 0 & 1 \\
0 & 0 & 0 & 0
\end{array}\right],
$$

$$
B=\left[\begin{array}{ccc}
\frac{\sin \delta}{I_{\alpha}} & \cdots & \frac{\sin N \delta}{I_{\alpha}} \\
0 & \cdots & 0 \\
\frac{\sin \delta}{I_{\beta}} & \cdots & \frac{\sin N \delta}{I_{\beta}}
\end{array}\right] .
$$

When there are random frictions caused by the load, we have

$$
\left[\begin{array}{c}
\dot{\varphi}_{\alpha} \\
\dot{\omega}_{\alpha} \\
\dot{\varphi}_{\beta} \\
\dot{\omega}_{\beta}
\end{array}\right]=A\left[\begin{array}{c}
\varphi_{\alpha} \\
\omega_{\alpha} \\
\varphi_{\beta} \\
\omega_{\beta}
\end{array}\right]+B\left(\left[\begin{array}{c}
F_{1}-\frac{G}{N} \\
\vdots \\
F_{N}-\frac{G}{N}
\end{array}\right]+\left[\begin{array}{c}
\xi_{1} \\
\vdots \\
\xi_{N}
\end{array}\right]\right),
$$

where $\left[\begin{array}{llll}\xi_{1} & \cdots & \xi_{N}\end{array}\right]^{T}$ is a random force vector.

In this extrusion machine case, denote $s_{i}$ as the position of the number $i$ cylinder's force point, and denote $v_{i}$ as the velocity of the number $i$ cylinder's force point. According to the conclusion in Appendix A, the observation matrix of the position is

$$
\left[\begin{array}{l}
S \\
V
\end{array}\right]=\left[\begin{array}{l}
C \varphi \\
C \omega
\end{array}\right]
$$

where $S=\left[\begin{array}{llllll}s_{1} & s_{2} & s_{3} & s_{4} & s_{5} & s_{6}\end{array}\right]^{T}, V=$ $\left[\begin{array}{llllll}v_{1} & v_{2} & v_{3} & v_{4} & v_{5} & v_{6}\end{array}\right]^{T}, \varphi=\left[\begin{array}{ll}\varphi_{\alpha} & \varphi_{\beta}\end{array}\right]^{T}, \omega=$ $\left[\begin{array}{ll}\omega_{\alpha} & \omega_{\beta}\end{array}\right]^{T}$, and

$$
C=r\left[\begin{array}{cccccc}
\frac{1}{2} & -\frac{1}{2} & 0 & -\frac{1}{2} & \frac{1}{2} & 0 \\
\frac{\sqrt{3}}{2} & \frac{\sqrt{3}}{2} & -1 & \frac{-\sqrt{3}}{2} & \frac{-\sqrt{3}}{2} & 1
\end{array}\right]^{T} .
$$




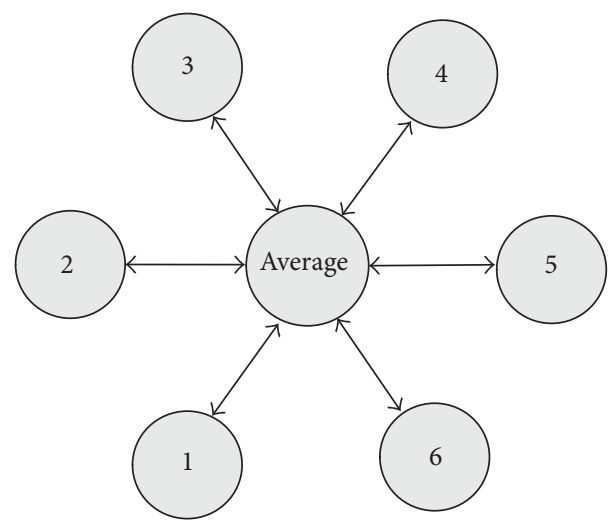

FIgURE 6: The graph used for consensus control.

\section{Control Strategy Design}

The control design problem is to design controller with low complexity to stabilize the main table described by (13) with available measurements given in (14).

3.1. Consensus Control Strategy Design. With the idea borrowed from the multiagent and consensus control which was briefly reviewed in Appendix B, we now try to design simple yet effective control strategies for our extrusion machine.

The forty-two pumps are divided into six groups, each one of which is assigned to one cylinder, so the cylinders are independent from each other. Then, we can regard the six cylinders as six agents, and they constitute a multiagent system, the topology of which is the physical constraint of the main table. Our purpose of control is maintaining the same velocity and position of the six cylinders during the extrusion process.

In fact, there are six hydraulic cylinders offering the force, and the topology of the agent network is shown in Figure 6. Our control protocol is

$$
\begin{aligned}
& F_{i}=u_{i}+\frac{G}{N}, \\
& u_{i}=u_{i 1}+u_{i 2},
\end{aligned}
$$

where

$$
\begin{aligned}
& u_{i 1}=-k_{i}\left(v_{i}-v_{0}\right), \\
& u_{i 2}=-a_{i}\left(s_{i}-\bar{s}\right),
\end{aligned}
$$

where $v_{0}$ is the target speed of the cylinders and $\bar{s}$ is the average position of the six cylinders.

Substituting into system equation (13) we can get

$$
\left[\begin{array}{c}
\dot{\varphi}_{\alpha} \\
\dot{\omega}_{\alpha} \\
\dot{\varphi}_{\beta} \\
\dot{\omega}_{\beta}
\end{array}\right]=A\left[\begin{array}{c}
\varphi_{\alpha} \\
\omega_{\alpha} \\
\varphi_{\beta} \\
\omega_{\beta}
\end{array}\right]+B\left(\left[\begin{array}{c}
-k_{1}\left(v_{1}-v_{0}\right) \\
-a_{1}\left(s_{1}-\bar{s}\right) \\
\vdots \\
-a_{N}\left(s_{N}-\bar{s}\right)
\end{array}\right]+\left[\begin{array}{c}
\xi_{1} \\
\xi_{2} \\
\vdots \\
\xi_{N}
\end{array}\right]\right) .
$$

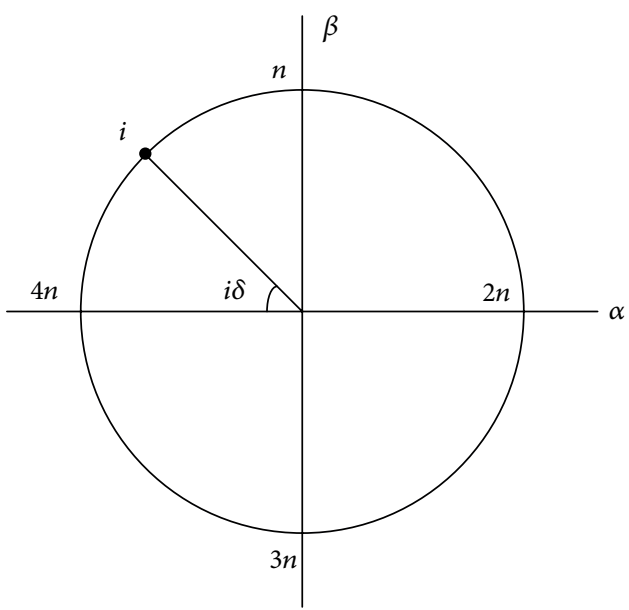

FIgURE 7: The circular plane case 1: $N=4 n$.

Remark 1. Although our control protocol looks similar to the existing ones, it should be pointed out that the dynamics of the overall system is more challenging than the systems in the consensus control literature. There, the state equations of the agents are independent. In contrast, in our system, the state equations of the agents are coupled. They have to satisfy the geometry constraint enforced by the main table. (See the geometric derivation in the appendix).

Remark 2. When replacing the $\bar{s}$ with the $s_{0}$, which means that the position is set in advance, the topology connection between the agents has been cut down, and the consensus control turns into independent loop PID (actually PI) control strategy. When we analyze the attitude of the plane model, because the form of the two control strategy is the same, the convergence condition and the numerical simulation results are the same too. But in the actual producing process, because the $\bar{s}$ is more flexible and more close to reality than $s_{0}$, the consensus control's effect is also better than the independent loop PID control's effect. (We will prove it in Section 6 in the means of cosimulation platform).

\section{The Convergence Condition Derivation}

In this section, we try to find the convergence condition of our control protocol in Section 3. Depending on the number of cylinders, we will discuss three cases, $N=4 n, N=4 n+2$, and $N=2 n+1$, for any integer $n$. We will derive our convergence conditions based directly on Routh stability criterion.

In general, due to the symmetry of the distribution of forces, the $G / N$ part of the $F_{i}$ is counteracting to the gravity each hydraulic cylinder subjected to. So we can assume a gravity-free environment, which means $F_{i}=u_{i}$, to deduce the convergence condition derivation.

Also, the random frictions are not taken into consideration during our derivation. 
Under these assumptions, we can get our convergence condition of the consensus control strategy as follows:

$$
\begin{aligned}
& \sum_{i=1}^{N} k_{i} \cos ^{2} i \delta>0, \quad \sum_{i=1}^{N} k_{i} \sin ^{2} i \delta>0, \\
& \sum_{i=1}^{N} a_{i} \cos ^{2} i \delta>0, \quad \sum_{i=1}^{N} a_{i} \sin ^{2} i \delta>0 .
\end{aligned}
$$

Next, we are going to prove this conclusion.

4.1. $N=4 n$ : Uniform Distribution. The distribution of the agents is both Centrosymmetric and Axisymmetric.

Assume point $2 n$ locates at the axis $\alpha$, the relative position between point $2 n$ and the center point is $s_{\alpha}$, and the velocity of the point is $v_{\alpha}$, and assume point $n$ locates at the axis $\beta$, the relative position between the point and the center point is $s_{\beta}$, and the velocity of the point is $v_{\beta}$. Without loss of generality, we can assume that $v_{\alpha}, s_{\beta}$ and $v_{\beta}$ are all greater than 0 (Figure 7).

$$
\left[\begin{array}{c}
\dot{\varphi}_{\alpha} \\
\dot{\omega}_{\alpha} \\
\dot{\varphi}_{\beta} \\
\dot{\omega}_{\beta}
\end{array}\right]=\left[\begin{array}{cc}
0 & 1 \\
-\sum_{i=1}^{N} a_{i} \sin ^{2} i \delta & -\sum_{i=1}^{N} k_{i} \sin ^{2} i \delta \\
0 & 0 \\
-\sum_{i=1}^{N} a_{i} \cos i \delta \sin i \delta & -\sum_{i=1}^{N} k_{i} \cos i \delta \sin i \delta
\end{array}\right.
$$

Assume

$$
\begin{gathered}
p=-\sum_{i=1}^{N} k_{i} \cos i \delta \sin i \delta, \\
p^{\prime}=-\sum_{i=1}^{N} a_{i} \cos i \delta \sin i \delta, \\
q=-\sum_{i=1}^{N} k_{i} \sin ^{2} i \delta, \\
q^{\prime}=-\sum_{i=1}^{N} a_{i} \sin ^{2} i \delta, \\
l=-\sum_{i=1}^{N} k_{i} \cos ^{2} i \delta, \\
l^{\prime}=-\sum_{i=1}^{N} a_{i} \cos ^{2} i \delta .
\end{gathered}
$$

So the coefficient matrix $A$ can be rewritten as follows:

$$
A=\left[\begin{array}{cccc}
0 & 1 & 0 & 0 \\
q^{\prime} & q & p^{\prime} & p \\
0 & 0 & 0 & 1 \\
p^{\prime} & p & l^{\prime} & l
\end{array}\right] .
$$

From the analysis in Appendix A, we know how to calculate the perpendicular distance between any edge point of the circular plane and the horizontal plane.

Assume the point $J$ is the highest point of all the edge points of the circular point from the horizontal plane, with $\angle A O J=\theta$, and the initial position is

$$
s_{i}=\cos (i \delta-\theta) \cdot \sqrt{s_{\alpha}^{2}+s_{\beta}^{2}}=s_{\alpha} \sin i \delta+s_{\beta} \cos i \delta,
$$

where $s_{\alpha}=\varphi_{\alpha} \cdot r, s_{\beta}=\varphi_{\beta} \cdot r$.

The velocity is the first derivative of the position, so we can get

$$
v_{i}=\frac{d\left(s_{\alpha} \cos (i \delta)+s_{\beta} \sin i \delta\right)}{d t}=v_{\alpha} \sin i \delta+v_{\beta} \cos i \delta,
$$

where $v_{\alpha}=\omega_{\alpha} \cdot r, v_{\beta}=\omega_{\beta} \cdot r$.

According to (18), we have

$$
\left.\begin{array}{cc}
-\sum_{i=1}^{N} a_{i} \cos i \delta \sin i \delta & -\sum_{i=1}^{N} k_{i} \cos i \delta \sin i \delta \\
0 & 1 \\
-\sum_{i=1}^{N} a_{i} \cos ^{2} i \delta & -\sum_{i=1}^{N} k_{i} \cos ^{2} i \delta
\end{array}\right]\left[\begin{array}{c}
\varphi_{\alpha} \\
\omega_{\alpha} \\
\varphi_{\beta} \\
\omega_{\beta}
\end{array}\right] .
$$

So

$$
\begin{aligned}
|s I-A|= & s^{4}-(q+l) s^{3}+\left(q l-q^{\prime}-l^{\prime}-p^{2}\right) s^{2} \\
& +\left(q^{\prime} l+q l^{\prime}-2 p p^{\prime}\right) s+\left(q^{\prime} l^{\prime}-p^{\prime 2}\right) \\
= & t_{0} s^{4}+t_{1} s^{3}+t_{2} s^{2}+t_{3} s+t_{4} .
\end{aligned}
$$

With the Routh stability criterion, which provides the necessary and sufficient conditions for the convergence of the polynomial, we can get

$$
\begin{gathered}
t_{0}, t_{1}, t_{2}, t_{3}, t_{4}>0, \\
t_{1} t_{2}-t_{0} t_{3}>0,
\end{gathered}
$$

$$
t_{3}\left(t_{1} t_{2}-t_{0} t_{3}\right)-t_{1}^{2} t_{4}>0
$$

Now, we are trying to find a set of solutions to the array of the inequalities above. 


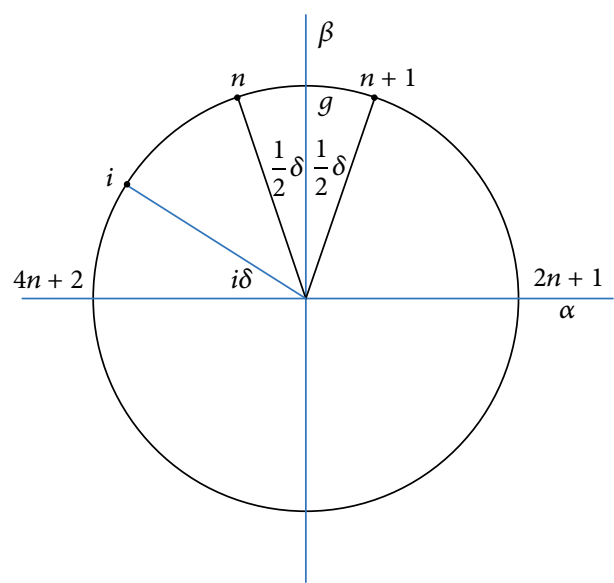

FIgURE 8: The circular plane case 1: $N=4 n+2$.

Assign $n=1$; then $N=4 n=4$. In this case

$$
\begin{gathered}
p=-\sum_{i=1}^{N} k_{i} \cos i \delta \sin i \delta=0, \\
p^{\prime}=-\sum_{i=1}^{N} a_{i} \cos i \delta \sin i \delta=0, \\
q=-\sum_{i=1}^{N} k_{i} \sin ^{2} i \delta=-\left(k_{2}+k_{4}\right), \\
q^{\prime}=-\sum_{i=1}^{N} a_{i} \sin ^{2} i \delta=-\left(a_{2}+a_{4}\right), \\
l=-\sum_{i=1}^{N} k_{i} \cos ^{2} i \delta=-\left(k_{1}+k_{3}\right), \\
l^{\prime}=-\sum_{i=1}^{N} a_{i} \cos ^{2} i \delta=-\left(a_{1}+a_{3}\right) .
\end{gathered}
$$

According to the inequalities array (26), we can get the result

$$
\begin{array}{ll}
k_{2}+k_{4}>0, & k_{1}+k_{3}>0, \\
a_{2}+a_{4}>0, & a_{1}+a_{3}>0 .
\end{array}
$$

These can meet the conclusion (19).

When $n>1$, each force $F_{i \delta}$ can be decomposed into two mutually perpendicular directions: axis $\alpha$ and axis $\beta$, and the coefficients are $\cos i \delta$ or $\sin i \delta$. And the total effect is just like the case when $n=1$; also, the calculation steps are the same with $n=1$.

So the conclusion (19) is the solution to the array of the inequalities (26) (Figure 8).

4.2. $N=4 n+2$ : Uniform Distribution. In this situation, the distribution of the agents is also Centrosymmetric and Axisymmetric. We cannot obtain $s_{\alpha}, s_{\beta}, v_{\alpha}, v_{\beta}$ (or say $\varphi_{\alpha}, \varphi_{\beta}$, $\left.\omega_{\alpha}, \omega_{\beta}\right)$ directly.

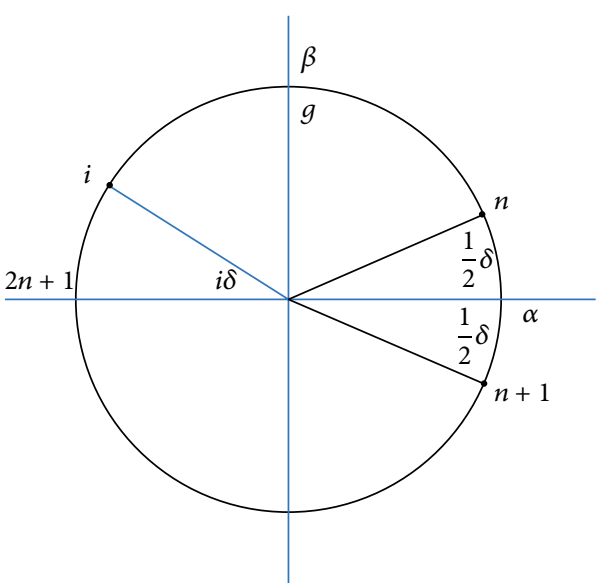

Figure 9: The circular plane case $1: N=2 n+1$.

Assume the $n$ point's relative deviation from the center point is $s_{1}$, the velocity of the point is $v_{1}$, the $n+1$ point's relative deviation from the center point is $s_{2}$, and the velocity of the point is $v_{2}$.

We can get $4 n+2$ (or say 0 ), which locate at the axis $\alpha$, and the point's relative deviation from the center point is $s_{\alpha}=$ $\left(s_{1}-s_{2}\right) / 2 \cos (\delta / 2)$, the velocity of the point is $v_{\alpha}=\left(v_{1}-\right.$ $\left.v_{2}\right) / 2 \cos (\delta / 2)$, and we can get the virtual point, which locates at the axis $\beta$, point's relative deviation from the center point is $s_{\beta}=\left(s_{1}+s_{2}\right) / 2 \sin (\delta / 2)$, and the velocity of the point is $v_{\beta}=\left(v_{1}+v_{2}\right) / 2 \sin (\delta / 2)$.

Then, the next steps are the same as the case $N=4 n$, and we can get the same equations (22), (25), and (26) too.

Assume $n=0$; then $N=4 n+2=2$. In this case

$$
\begin{gathered}
p=-\sum_{i=1}^{N} k_{i} \cos i \delta \sin i \delta=0, \\
p^{\prime}=-\sum_{i=1}^{N} a_{i} \cos i \delta \sin i \delta=0, \\
q=-\sum_{i=1}^{N} k_{i} \sin ^{2} i \delta=0, \\
q^{\prime}=-\sum_{i=1}^{N} a_{i} \sin ^{2} i \delta=0, \\
l=-\sum_{i=1}^{N} k_{i} \cos ^{2} i \delta=-\left(k_{1}+k_{2}\right), \\
l^{\prime}=-\sum_{i=1}^{N} a_{i} \cos ^{2} i \delta=-\left(a_{1}+a_{2}\right) .
\end{gathered}
$$

According to the inequalities array, we can get the result

$$
k_{1}+k_{2}>0, \quad a_{1}+a_{2}>0 .
$$

These can meet the conclusion (19).

When $n>0$, each force $F_{i \delta}$ can be decomposed into two mutually perpendicular directions: axis $\alpha$ and axis $\beta$, and 


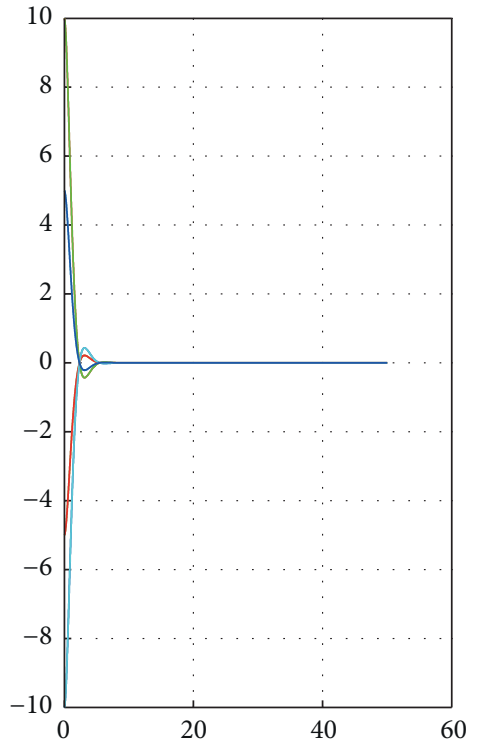

(a)

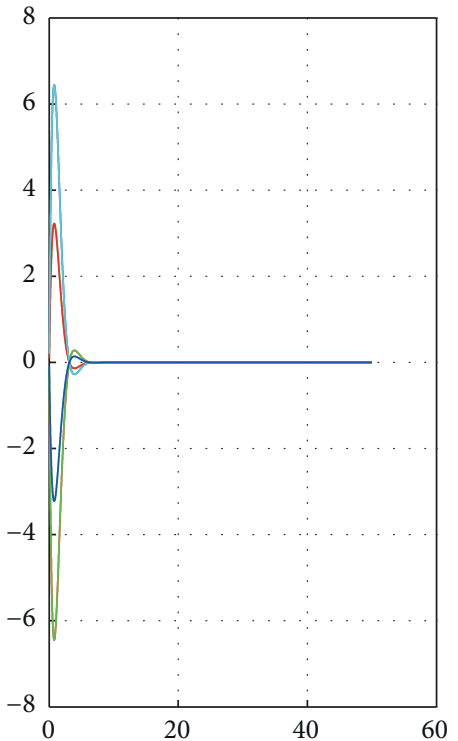

(b)

FIGURE 10: (a) is the position of each agent; (b) is the velocity of each agent.

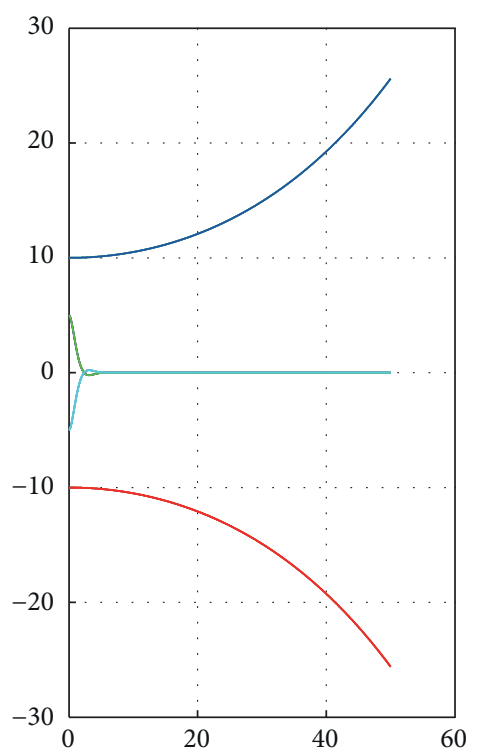

(a)

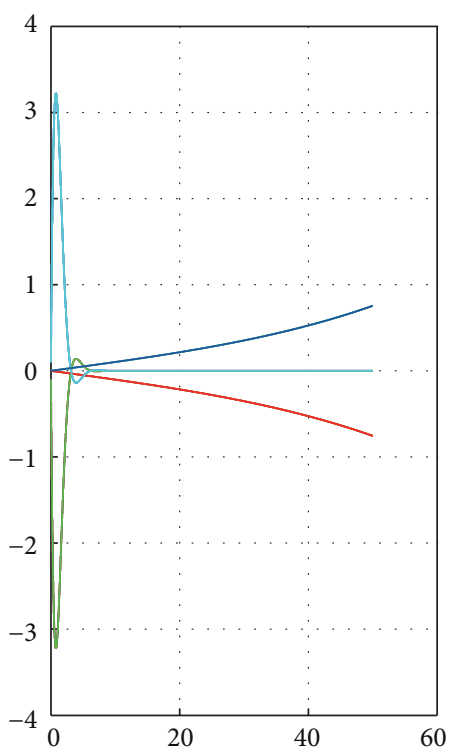

(b)

FIGURE 11: (a) is the position of each agent; (b) is the velocity of each agent.

the coefficients are $\cos i \delta$ or $\sin i \delta$. And the total effect is just like the case when $N=4$; also, the calculation steps are the same with $N=4$.

So the conclusion (19) is the convergence condition of this case.

4.3. $N=2 n+1$ : Uniform Distribution. In this situation, the distribution of the agents is Axisymmetric. We cannot obtain $s_{\alpha}, s_{\beta}, v_{\alpha}, v_{\beta}$ (or say $\varphi_{\alpha}, \varphi_{\beta}, \omega_{\alpha}, \omega_{\beta}$ ) directly either.
Assume the $n$ point's relative deviation from the center point is $s_{1}$ and the velocity of the point is $v_{1}$, and assume the $n+1$ point's relative deviation from the center point is $s_{2}$ and the velocity of the point is $v_{2}$.

And we can get $2 n+1$ (or say 0 ), which locate at the axis $\alpha$, point's relative deviation from the center point is $s_{\alpha}=\left(s_{1}+s_{2}\right) / 2 \sin (\delta / 2)$, and the velocity of the point is $v_{\alpha}=\left(v_{1}+v_{2}\right) / 2 \sin (\delta / 2)$, and we can get the virtual point, which locates at the axis $\beta$, point's relative deviation from 


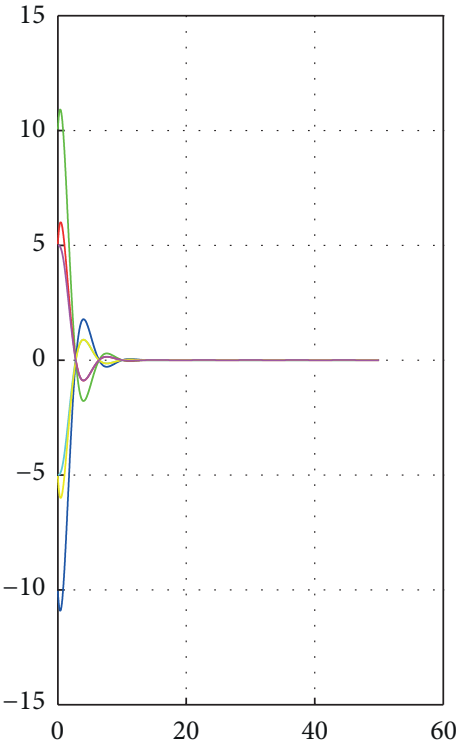

(a)

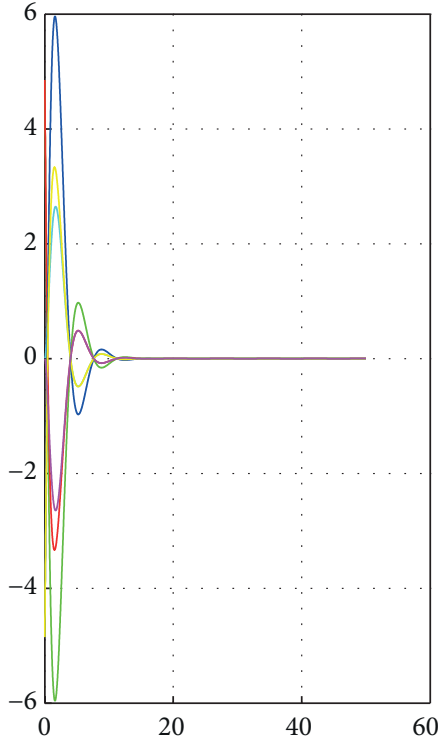

(b)

FIGURE 12: (a) is the position of each agent; (b) is the velocity of each agent.

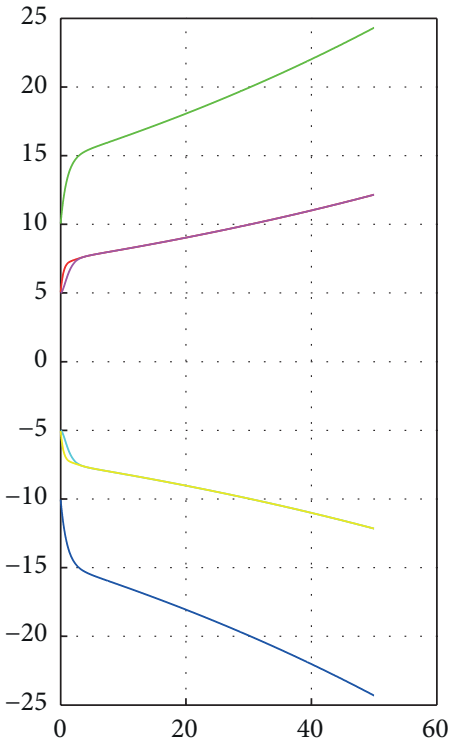

(a)

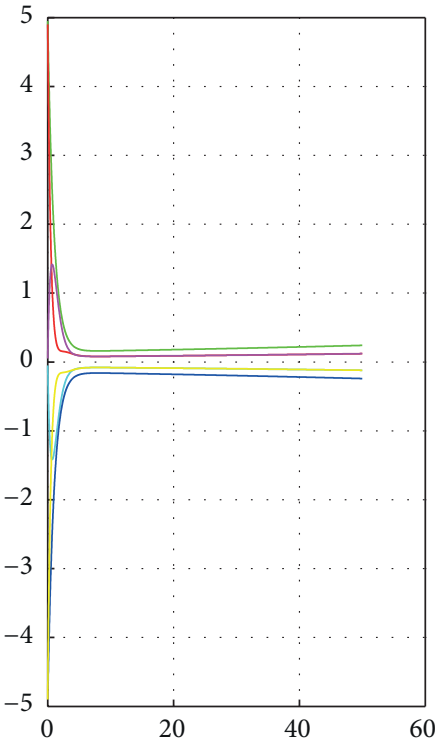

(b)

FIGURE 13: (a) is the position of each agent; (b) is the velocity of each agent.

the center point is $s_{\beta}=\left(s_{1}-s_{2}\right) / 2 \cos (\delta / 2)$, and the velocity of the point is $v_{\alpha}=\left(v_{1}-v_{2}\right) / 2 \cos (\delta / 2)$ (Figure 9$)$.

Then, the next steps are the same as the case of $N=4 n$, and we can get the same equations (22), (25), and (26) too. Assuming $n=0$, then $N=2 n+1=1$. In this case

$$
\begin{aligned}
& p=-\sum_{i=1}^{N} k_{i} \cos i \delta \sin i \delta=0, \\
& p^{\prime}=-\sum_{i=1}^{N} a_{i} \cos i \delta \sin i \delta=0,
\end{aligned}
$$

$$
\begin{gathered}
q=-\sum_{i=1}^{N} k_{i} \sin ^{2} i \delta=0, \\
q^{\prime}=-\sum_{i=1}^{N} a_{i} \sin ^{2} i \delta=0, \\
l=-\sum_{i=1}^{N} k_{i} \cos ^{2} i \delta=-k_{1}, \\
l^{\prime}=-\sum_{i=1}^{N} a_{i} \cos ^{2} i \delta=-a_{1} .
\end{gathered}
$$


According to the inequalities array, we can get the result

$$
k_{1}>0, \quad a_{1}>0 .
$$

These can meet the conclusion (19).

When $n>0$, each force $F_{i \delta}$ can be decomposed into two mutually perpendicular directions: axis $\alpha$ and axis $\beta$, and the coefficients are $\cos i \delta$ or $\sin i \delta$. And the total effect is just like the case when $N=4$; also, the calculation steps are the same with $N=4$.

So the conclusion (19) is the convergence condition of this case.

Above all, we have proved that the conclusion (19) is the convergence condition of our consensus control strategy.

\section{Simple Mathematical Simulation}

In this section, we do some simulation to illustrate the control design and convergence analysis. We will focus on the specific examples; we will do the simulation on the case in which $N=$ 4 and $N=6$.

5.1. Square Case. In this section, there are four agents that need to be controlled, and each of them has two variables: the position and the velocity. The initial value of each variable is set as follows:

$$
\begin{gathered}
s_{1}=10, \quad s_{2}=5, \\
s_{3}=-10, \quad s_{4}=-5, \\
v_{1}=v_{2}=v_{3}=v_{4}=0 .
\end{gathered}
$$

At first, all the feedback coefficients are set equal to 1 , which means $k_{1}=k_{2}=k_{3}=k_{4}=1$ and $a_{1}=a_{2}=a_{3}=a_{4}=1$.

The simulation results of the position and velocity of each agent are shown in Figure 10. We can learn the convergence time is about $10 \mathrm{~s}$.

Then, let us carry out simulations for parameters violating the convergence conditions established in Section 6. We set the coefficients as follows:

$$
\begin{array}{ll}
k_{1}=-1.001, & k_{2}=k_{3}=k_{4}=1, \\
a_{1}=-1.001, & a_{2}=a_{3}=a_{4}=1,
\end{array}
$$

and check the position and velocity of each agent. We can see from Figure 11 that, as expected, the system could not keep convergence any more. Moreover, it is the axis $k_{1}+k_{3}>0$, $a_{1}+a_{3}>0$ that could not converge, while the other axis still meets the convergence requirement.

5.2. The Hexagon Case. We design the simulation as the case of square.

The initial value of each variable is set as

$$
\begin{array}{ccc}
s_{1}=10, & s_{2}=5, & s_{3}=-5, \\
s_{4}=-10, & s_{5}=-5, & s_{6}=5, \\
v_{1}=5, & v_{2}=5, & v_{3}=0, \\
v_{4}=-5, & v_{5}=-5, & v_{6}=0 .
\end{array}
$$

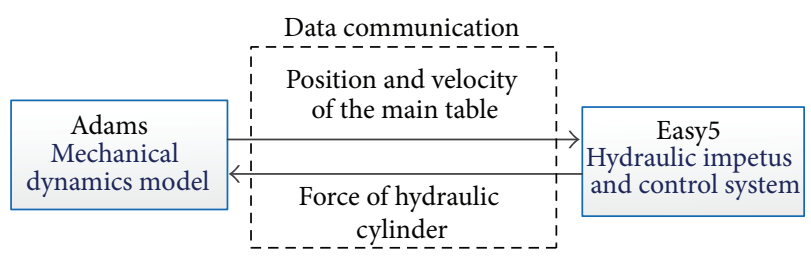

Figure 14: The cosimulation of Adams and Easy5.

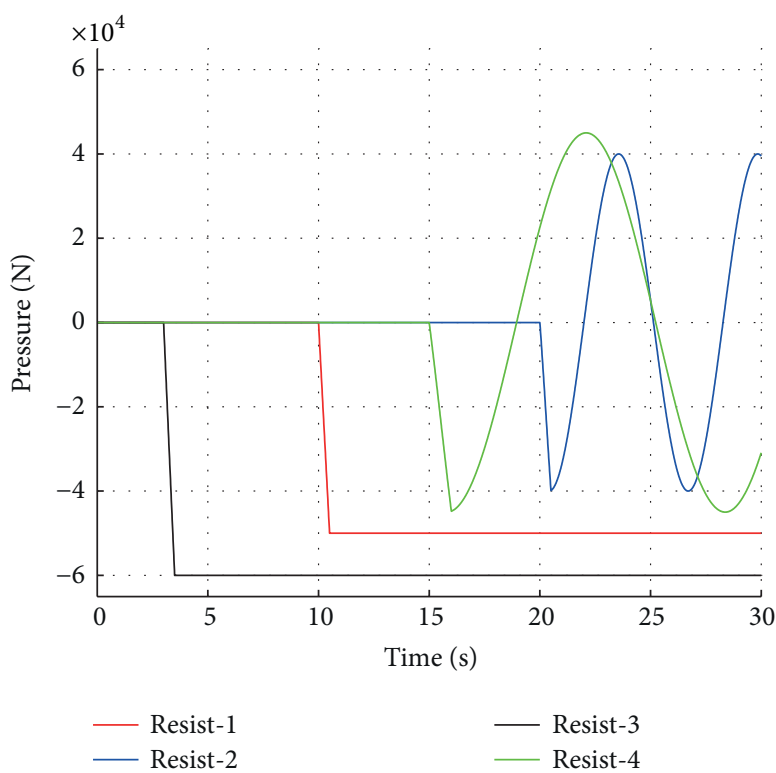

FIGURE 15: The resistance forces in the simulation.

At first, we set the velocity feedback coefficients $k_{1}=k_{2}=$ $k_{3}=k_{4}=k_{5}=k_{6}=1$ and the position feedback coefficients $a_{1}=a_{2}=a_{3}=a_{4}=a_{5}=a_{6}=1$. The simulation results of the position and velocity of each agent are shown in Figure 12. We can learn the convergence time is about $20 \mathrm{~s}$.

Then, we check the situations where the convergence conditions are not satisfied. To be more specific, we run simulation for the case where

$$
\begin{array}{ll}
k_{1}=-1.001, & k_{2}=k_{3}=k_{4}=k_{5}=k_{6}=1, \\
a_{1}=-2.03, & a_{2}=a_{3}=a_{4}=a_{5}=a_{6}=1 .
\end{array}
$$

We check the position and velocity of each agent. As we can see from Figure 13, the system could not keep convergence any more.

\section{Simulation on the Cosimulation Platform}

One of the biggest differences between the mathematic simulation and the reality is that the simulation situation 


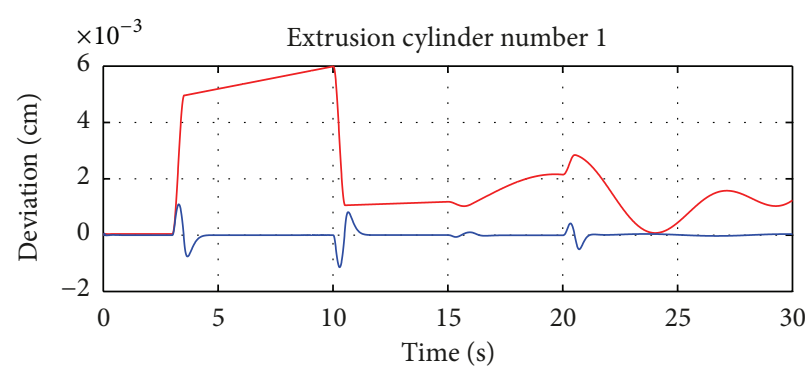

(a)

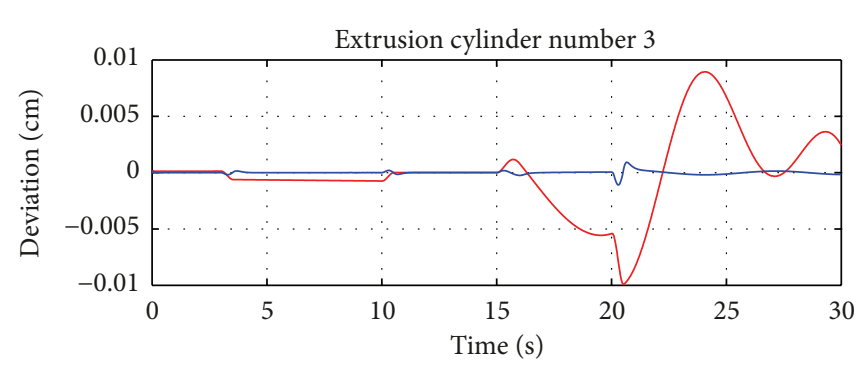

(c)

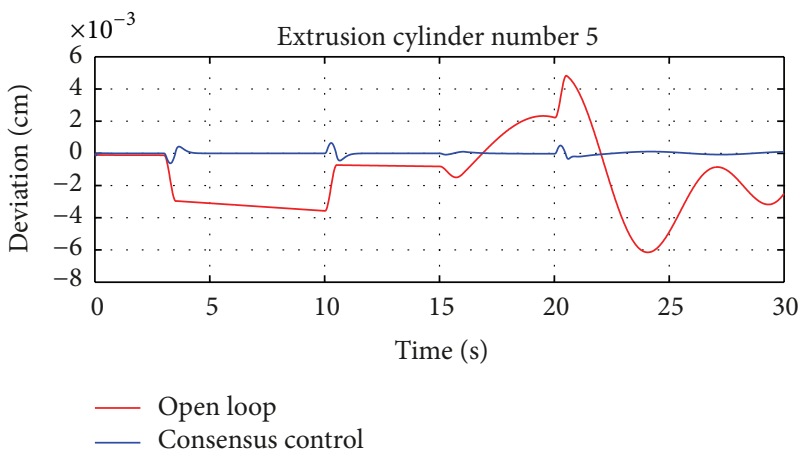

(e)

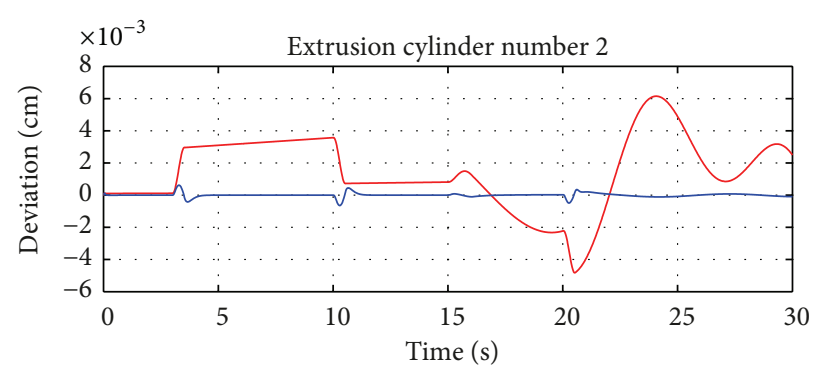

(b)

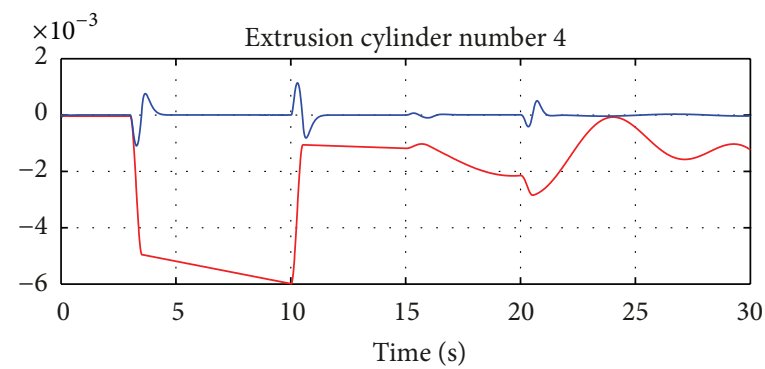

(d)

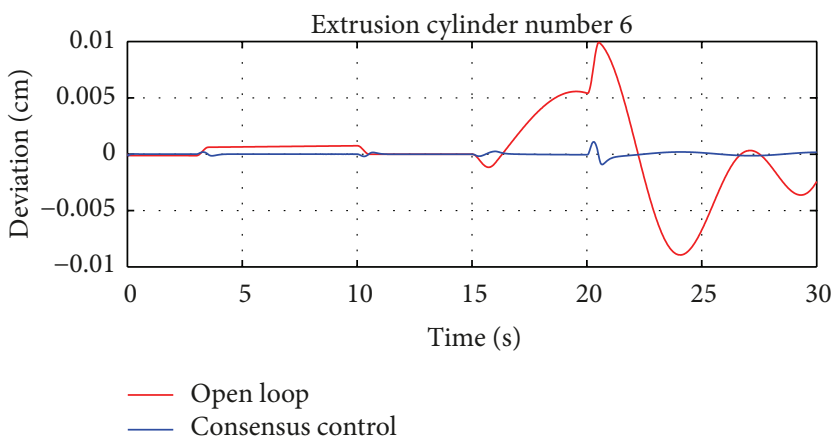

(f)

FIGURE 16: The position error of each cylinder.

cannot take the unforeseen circumstances into consideration. Factors like the temperature variation, measurement error, and feedback time delay are ignored while quite usual in the practice.

To make the simulation more realistic, we built a cosimulation platform consisting of Easy5 and Adams [12]. In this platform, Adams and Easy5 can exchange data to each other, while solving their own problems. The cosimulation principle is shown in Figure 14. Easy5 uses mechanical dynamic parameters as its input, simulates the responses of hydraulic system, and passes the results to Adams; Adams adopts hydraulic power parameters as its input, computes the responses of mechanical system, and delivers the consequences to Easy5. The communication of these two software programs uses Virtual Server-Client way to transfer data at settable step. The characteristics including validity and authenticity of the cosimulation platform have been proved in [12], and we will use this cosimulation platform to compare our control strategy with open loop control used currently in practice. In the following simulation, the period time of data exchanging between Easy5 and Adams is $0.01 \mathrm{~s}$.

6.1. Without Loading. In this case, we compare the open loop control and the consensus control strategy on the cosimulation platform without load. The resistance forces applied on the four corners are shown in Figure 15. The patterns of the disturbances include constant step functions and Sine functions. Consensus protocol used in this simulation is (17), with target speed of cylinders $v_{0}=5 \mathrm{~cm} / \mathrm{s}$; all the feedback coefficients are equal in groups $k_{1}=k_{2}=k_{3}=k_{4}=k_{5}=$ $k_{6}=150$ and all the feedback coefficients are equal in groups $a_{1}=a_{2}=a_{3}=a_{4}=a_{5}=a_{6}=400$. The simulation results are shown in Figure 16.

Table 1 lists the maximal position error and velocity error of each cylinder, and the bold ones of each column represent maximal position errors or velocity errors of all six cylinders. Form Figures 16 and 17 and Table 1, we can easily find that 


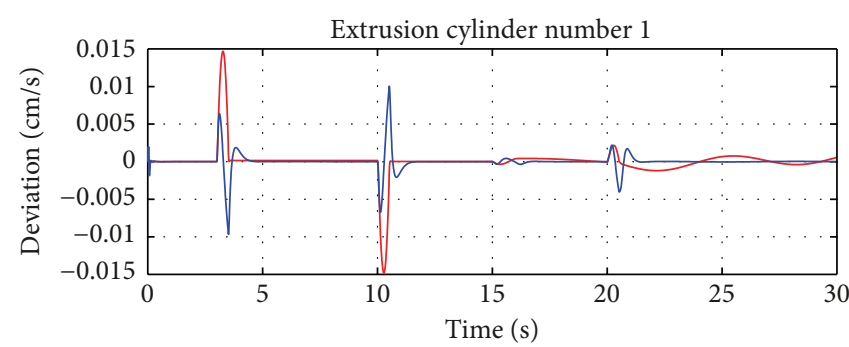

(a)

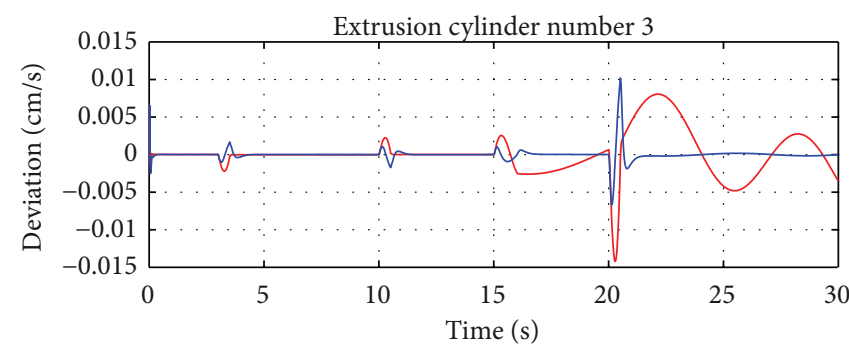

(c)

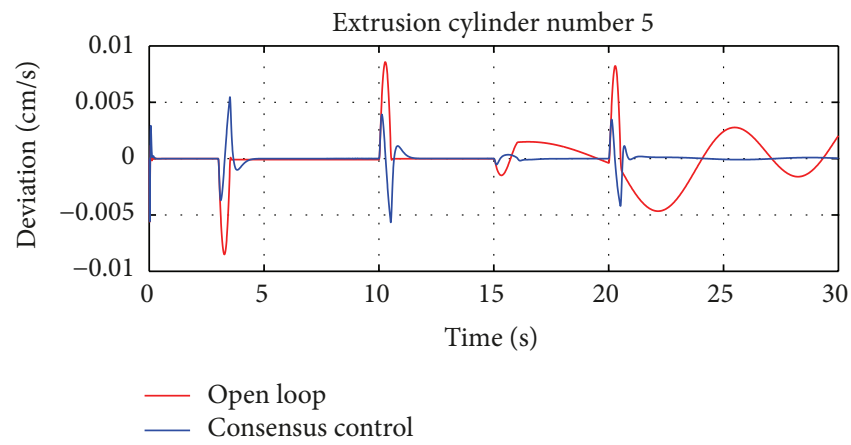

(e)

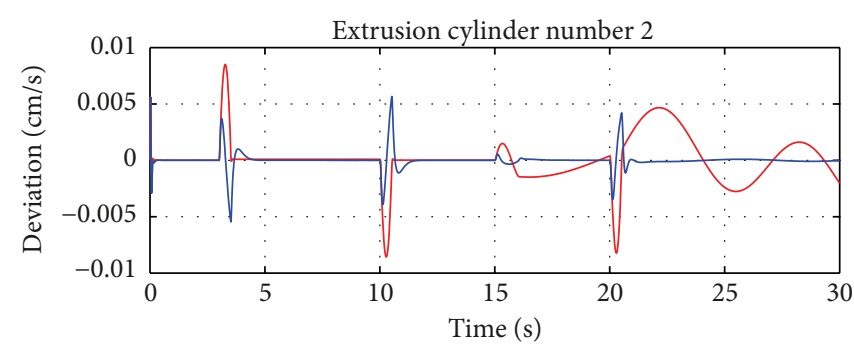

(b)

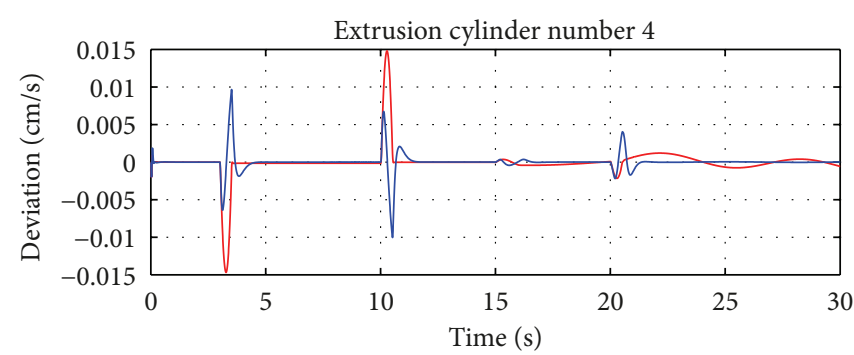

(d)

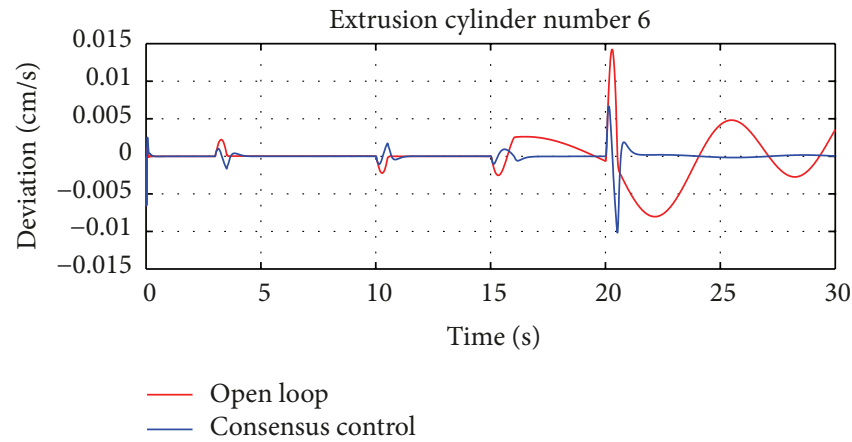

(f)

FIGURE 17: The velocity error of each cylinder.

the consensus control strategy has much better performance than the open loop control; in fact the maximum position error is reduced by one order of magnitude.

6.2. With Loading. In this case, we compare the open loop control and the consensus control strategy on the cosimulation platform with load. The load curve is shown in Figure 18. All the other settings, including period time, resistance, and control protocol, are the same as in the previous case. The simulation results are shown in Figure 17.

Table 2 lists the maximal position error and velocity error of each cylinder, and the bold ones of each column represent maximal position errors or velocity errors of all six cylinders. Form Figures 19 and 20 and Table 2, we can see that the consensus control strategy has much better performance than the open loop control when the system is loaded; the maximal position error is reduced more than 10 times.

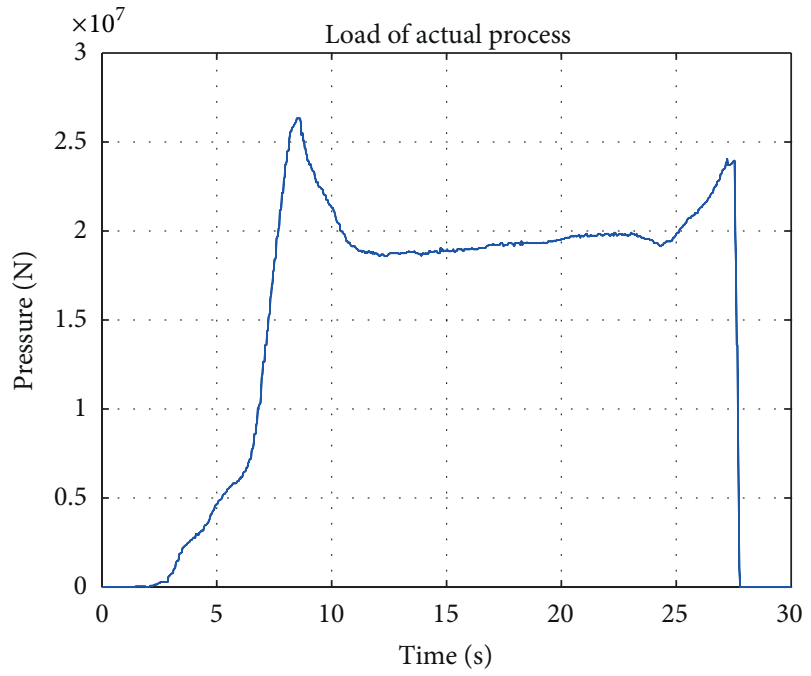

FIgURE 18: The load in the simulation. 


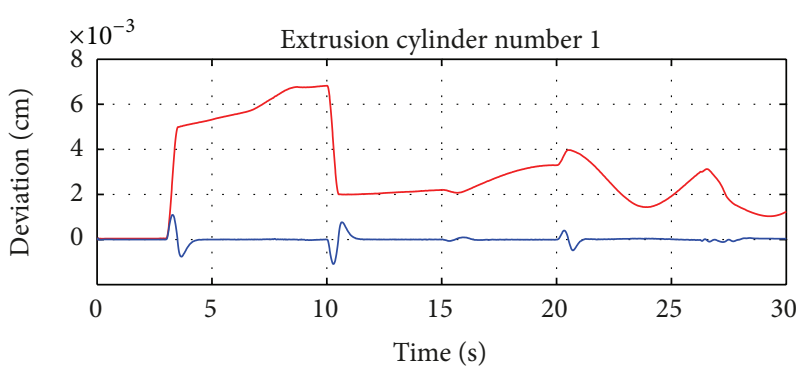

(a)

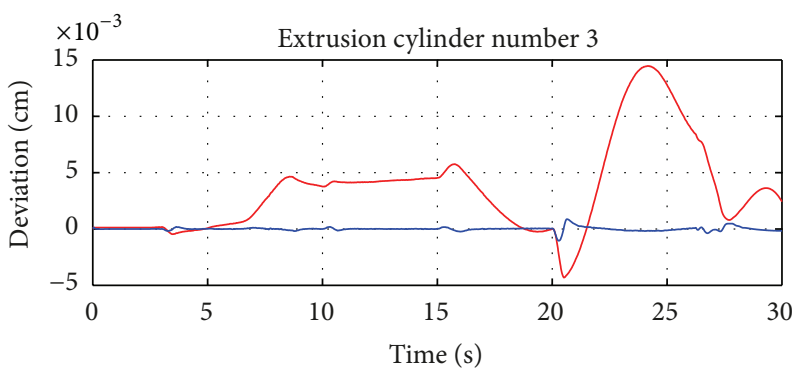

(c)

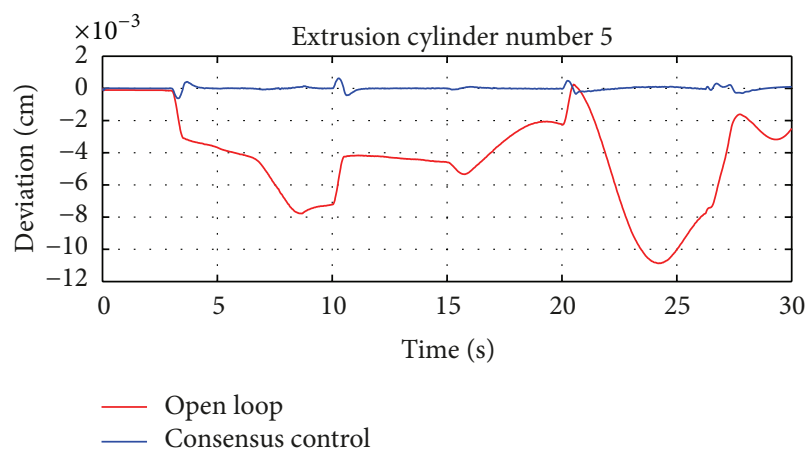

(e)

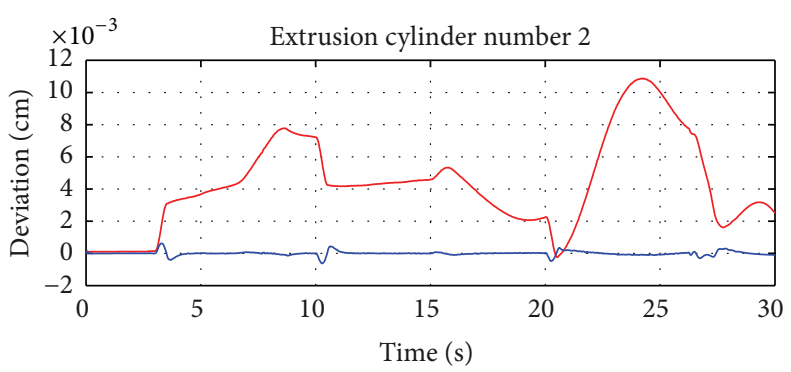

(b)

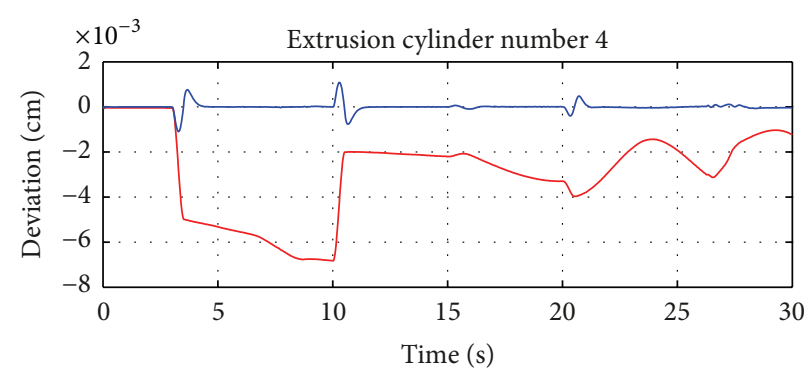

(d)

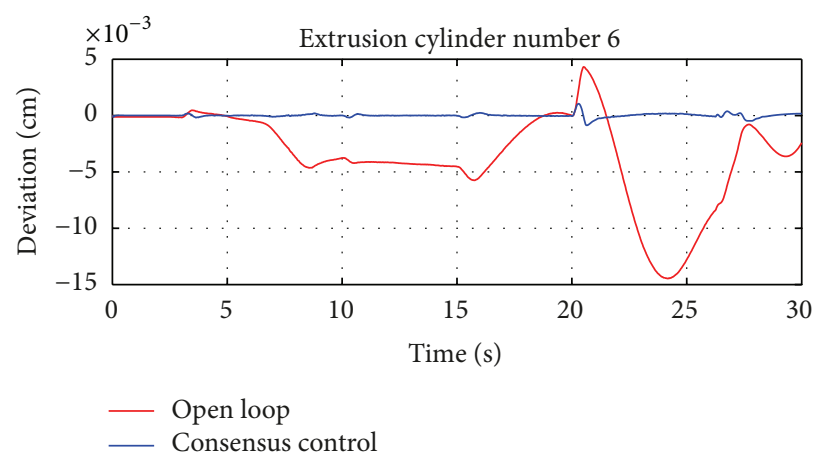

(f)

FIGURE 19: The position error of each cylinder.

TABLE 1: Maximal position error and velocity error of each cylinder.

\begin{tabular}{lcccc}
\hline Item & \multicolumn{2}{c}{ Position error $(\mathrm{cm})$} & \multicolumn{2}{c}{ Velocity error $(\mathrm{cm} / \mathrm{s})$} \\
Protocol & Open loop & Consensus & Open loop & Consensus \\
\hline 1 & 0.0060 & $\mathbf{0 . 0 0 1 1}$ & $\mathbf{0 . 0 1 4 8}$ & 0.0100 \\
2 & 0.0062 & 0.0006 & 0.0086 & 0.0057 \\
3 & $\mathbf{0 . 0 0 9 9}$ & $\mathbf{0 . 0 0 1 1}$ & 0.0142 & $\mathbf{0 . 0 1 0 2}$ \\
4 & 0.0060 & $\mathbf{0 . 0 0 1 1}$ & $\mathbf{0 . 0 1 4 8}$ & 0.0100 \\
5 & 0.0062 & 0.0006 & 0.0086 & 0.0057 \\
6 & $\mathbf{0 . 0 0 9 9}$ & $\mathbf{0 . 0 0 1 1}$ & 0.0142 & $\mathbf{0 . 0 1 0 2}$ \\
\hline
\end{tabular}

\section{Conclusions}

In previous practices, because of the strong coupling problem, the $360 \mathrm{MN}$ extrusion machine usually works under open loop control strategy. To improve its security and performance, we build a simplified control model for
TABLE 2: Maximal position error and velocity error of each cylinder.

\begin{tabular}{lcccc}
\hline Item & \multicolumn{2}{c}{ Position error $(\mathrm{cm})$} & \multicolumn{2}{c}{ Velocity error $(\mathrm{cm} / \mathrm{s})$} \\
Protocol & Open loop & Consensus & Open loop & Consensus \\
\hline 1 & 0.0068 & $\mathbf{0 . 0 0 1 1}$ & $\mathbf{0 . 0 1 4 7}$ & 0.0097 \\
2 & 0.0109 & 0.0006 & 0.0090 & 0.0056 \\
3 & $\mathbf{0 . 0 1 4 5}$ & 0.0010 & 0.0141 & $\mathbf{0 . 0 0 9 8}$ \\
4 & 0.0068 & $\mathbf{0 . 0 0 1 1}$ & $\mathbf{0 . 0 1 4 7}$ & 0.0097 \\
5 & 0.0109 & 0.0006 & 0.0090 & 0.0056 \\
6 & $\mathbf{0 . 0 1 4 5}$ & 0.0010 & 0.0141 & $\mathbf{0 . 0 0 9 8}$ \\
\hline
\end{tabular}

the $360 \mathrm{MN}$ extrusion machine and design a consensus control strategy to keep the main table work in a balanced attitude. Necessary and sufficient conditions are established for the convergence of the proposed control strategy based on the Routh stability criterion. The control strategy is also tested and compared with the open loop control strategy on 


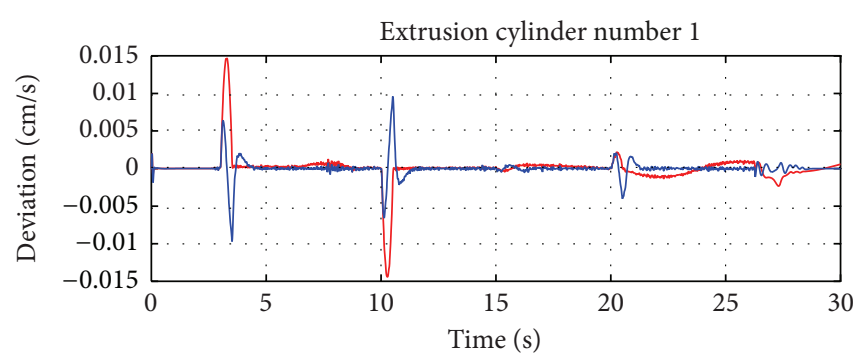

(a)

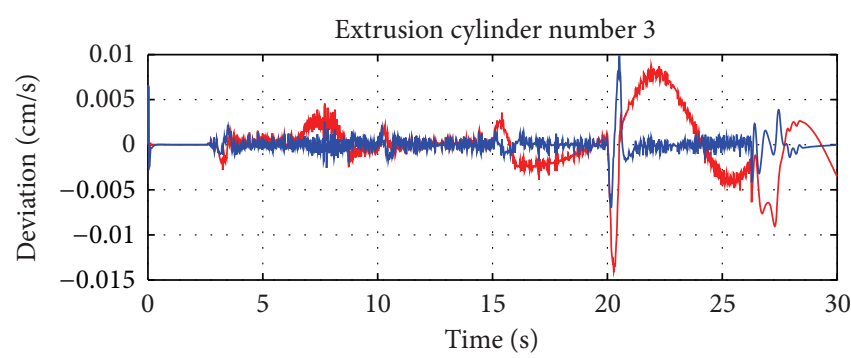

(c)

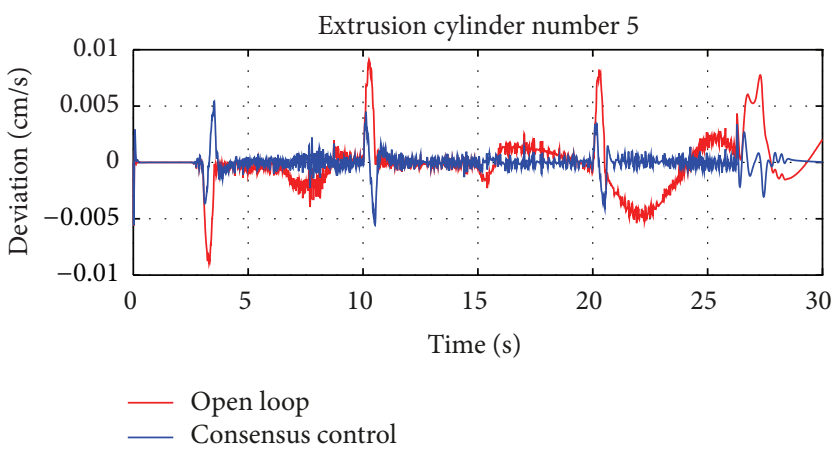

(e)

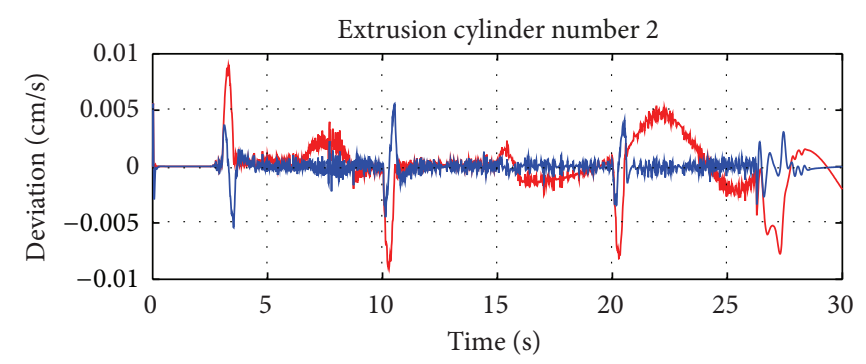

(b)

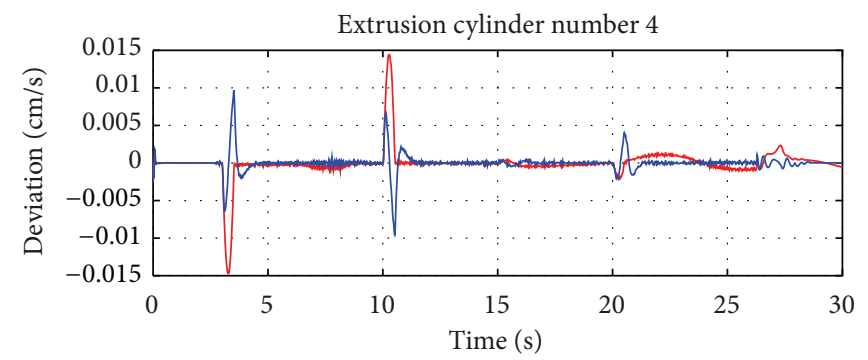

(d)

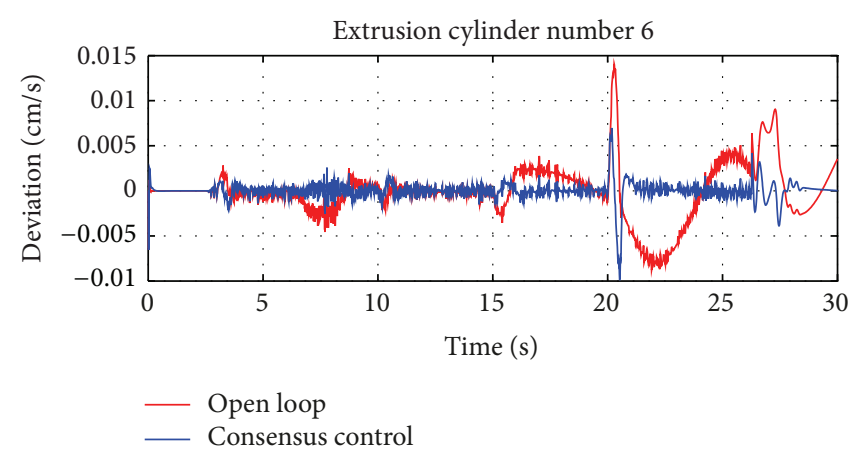

(f)

Figure 20: The velocity error of each cylinder.

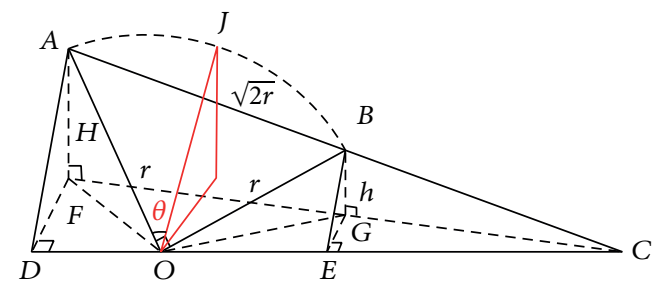

FIGURE 21: Solid geometric auxiliary figure.

a cosimulation platform. The results suggest that the consensus control method is a more effective way for the industrial process control problem.

Future work will emphasize more the application of the consensus like control strategy in reality; we will try to apply the model to the control system of $360 \mathrm{MN}$ vertical extrusion machine in Baotou. We will also deepen theoretical analysis of the problem to include more realistic situations (e.g., effects of limited communication rate similar to [13]).

\section{Appendices}

\section{A. Attitude Analysis of Circular Plane}

As we can see from Figure 21, the plane FCD is a horizontal plane and line $O A$ and line $O B$ are the two mutually perpendicular axes of the circular plane $O A B$. The distances between the horizontal plane and point $A$ and point $B$ are $H$ and $h$. Draw the extension line of $A B$ and meet the horizontal plane at point $C$. Connect the point $O$ and point $C$ then extended to point $D$. Draw the vertical line of point $A$ and point $B$ to line $C D$ at the point $D$ and point $E$.

As it is easy to know that the $\triangle A O D$ and $\triangle B O E$ are congruent and $\triangle C A D$ is similar to $\triangle C B E, \triangle C A F$ and $\triangle C B G$ are also similar. So we can get

$$
\frac{A D}{B E}=\frac{A C}{B C}=\frac{A F}{B G}=\frac{H}{h} .
$$

As $B E=\sqrt{r^{2}-O E^{2}}=\sqrt{r^{2}-A D^{2}}$, we have $A D /$ $\sqrt{r^{2}-A D^{2}}=H / h$. 
And the intersection angle between the circular plane and the horizontal plane is $\arcsin A F / A D=\arcsin \sqrt{H^{2}+h^{2}} / r$; the vertical distance is $\sqrt{H^{2}+h^{2}}$.

Because

$$
\cos \angle A O J=\sin \angle A O D=\frac{A D}{A O}=\frac{H}{\sqrt{H^{2}+h^{2}}},
$$

then

$$
\angle A O J=\arccos \frac{H}{\sqrt{H^{2}+h^{2}}} .
$$

So the perpendicular distance between any of the radiuses of the circular plane which has an intersection angle to the line $A O$ is $\cos (\angle A O J-\theta) \times \sqrt{H^{2}+h^{2}}$.

\section{B. Brief Review of Consensus Control Strategies}

Consensus control strategy was first introduced in the multiagent field [14]. The agent dynamics is adopted as a typical point mass model based on Newton's law. The average consensus problem is proposed for such class of networks [15], which includes two aspects, the agreement of the states of the agents and the convergence to zero of the speeds of the agents. A linear consensus protocol for such networks is established to solve such a consensus problem consisting of two parts, a local speed feedback controller and the interactions from the finite neighbors:

$$
\dot{s}_{i}=v_{i}, \quad m \dot{v}_{i}=u_{i}
$$

A linear control protocol can resolve the aforementioned average consensus problem. The control input can be calculated as follows:

$$
u_{i}=u_{i 1}+u_{i 2}
$$

where

$$
u_{i 1}=k v_{i}
$$

is the local speed feedback of each agent. The feedback gain $k$ will be illustrated later. Consider

$$
u_{i 2}=\sum_{j \in N_{i}} a_{i j}\left(s_{j}-s_{i}\right)
$$

is the part corresponding to the neighbors of node $\gamma_{i}$ which is constant in networks with fixed topology. In this paper, $u_{i 2}$ reflects the differences between a certain agent and its neighbor.

By using the above protocol, the agent dynamic is given as follows:

$$
\begin{gathered}
\dot{u}_{i}=v_{i} \\
m \dot{v}_{i}=k v_{i}+\sum_{j \in N_{i}} a_{i j}\left(s_{j}-s_{i}\right) .
\end{gathered}
$$

Specify $\varepsilon_{i}=\left[s_{i}, s_{j}\right]^{T}, i \in \delta$. Assume $m=1$; then we have

$$
\dot{\varepsilon}_{i}=A \varepsilon_{i}+B K \varepsilon_{i}+B F \sum_{j \in N_{i}} a_{i j}\left(s_{j}-s_{i}\right), \quad i \in \delta,
$$

where

$$
\begin{array}{cc}
A=\left[\begin{array}{ll}
0 & 1 \\
0 & 0
\end{array}\right], & B=\left[\begin{array}{l}
0 \\
1
\end{array}\right], \\
K=\left[\begin{array}{ll}
0 & k
\end{array}\right], & F=\left[\begin{array}{ll}
1 & 0
\end{array}\right] .
\end{array}
$$

Furthermore, construct $\varepsilon=\left[\begin{array}{llll}\varepsilon_{1} & \varepsilon_{2} & \cdots & \varepsilon_{M}\end{array}\right]^{T}$. The network dynamic is summarized as follows:

$$
\dot{\varepsilon}(t)=\tau \varepsilon(t),
$$

where

$$
\tau=I_{M} \cdot(A+B K)-L \cdot B F
$$

with $L$ being the Laplacian associated with the graph at the time [14].

\section{Conflict of Interests}

The authors declare that there is no conflict of interests regarding the publication of this paper.

\section{Acknowledgments}

The authors would like to thank the editor and the anonymous reviewers for very helpful comments to improve the quality of this paper. This work was supported by the National Natural Science Foundation of China (nos. 61174105, 61074034, 61021063, and 61174072). This paper was not presented at any IFAC meeting.

\section{References}

[1] B. R. Tibbetts and J. T.-Y. Wen, "Extrusion process control: mdeling, identification, and optimization," IEEE Transactions on Control Systems Technology, vol. 6, no. 2, pp. 134-145, 1998.

[2] Y. Yang, W. Yang, and H. Zhong, "Temperature distribution measurement and control of extrusion process by tomography," in Proceedings of the IEEE Workshop on Imaging Systems and Techniques (IST '08), pp. 170-174, grc, September 2008.

[3] M. H. B. DeGroot, "Reaching a consensus," Journal of the American Statistical Association, vol. 69, no. 345, pp. 118-121, 1974.

[4] R. Olfati-Saber, J. A. Fax, and R. M. Murray, "Consensus and cooperation in networked multi-agent systems," Proceedings of the IEEE, vol. 95, no. 1, pp. 215-233, 2007.

[5] R. Olfati-Saber, "Flocking for multi-agent dynamic systems: algorithms and theory," IEEE Transactions on Automatic Control, vol. 51, no. 3, pp. 401-420, 2006.

[6] L. Moreau, "Stability of multiagent systems with timedependent communication links," IEEE Transactions on Automatic Control, vol. 50, no. 2, pp. 169-182, 2005.

[7] L. Xiao and S. Boyd, "Fast linear iterations for distributed averaging," Systems and Control Letters, vol. 53, no. 1, pp. 65-78, 2004. 
[8] Y.-H. Chang, C.-W. Chang, and W.-C. Chan, "Fuzzy slidingmode consensus control for multi-agent systems," in Proceedings of the American Control Conference (ACC '11), pp. 16361641, July 2011.

[9] T. Namerikawa and C. Yoshioka, "Consensus control of observer-based multi-agent system with communication delay," in Proceedings of the International Conference on Instrumentation, Control and Information Technology (SICE '08), pp. 24142416, August 2008.

[10] G.-X. Wen and C. L. P. Chen, "Distributed consensus control using neural network for a class of nonlinear multi-agent systems," Systems, Man, and Cybernetics, pp. 2591-2595, 2012.

[11] W. Ren, H. Chao, W. Bourgeous, N. Sorensen, and Y. Chen, "Experimental validation of consensus algorithms for multivehicle cooperative control," IEEE Transactions on Control Systems Technology, vol. 16, no. 4, pp. 745-752, 2008.

[12] W. Li, W. Li, F. Peng, and Q. Zhao, "Control system simulation for 360MN extrusion machine based on physical model," in Proceedings of the 8th annual IEEE International Conference on Automation Science and Engineering, pp. 239-245, August 2012.

[13] T. Li, M. Fu, L. Xie, and J.-F. Zhang, "Distributed consensus with limited communication data rate," IEEE Transactions on Automatic Control, vol. 56, no. 2, pp. 279-292, 2011.

[14] G. Xie and L. Wang, "Consensus control for a class of networks of dynamic agents," International Journal of Robust and Nonlinear Control, vol. 17, no. 10-11, pp. 941-959, 2007.

[15] N. A. Lynch, Distributed Algorithms, Morgan Kaufmann, San Mateo, Calif, USA, 1997. 


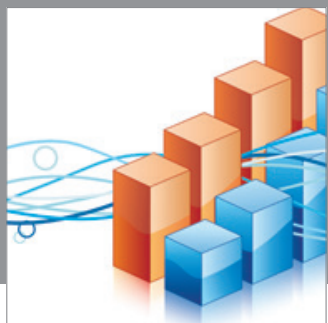

Advances in

Operations Research

mansans

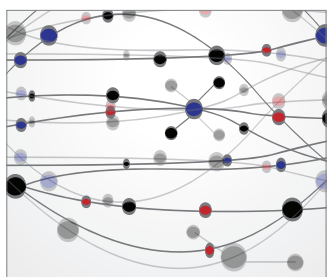

The Scientific World Journal
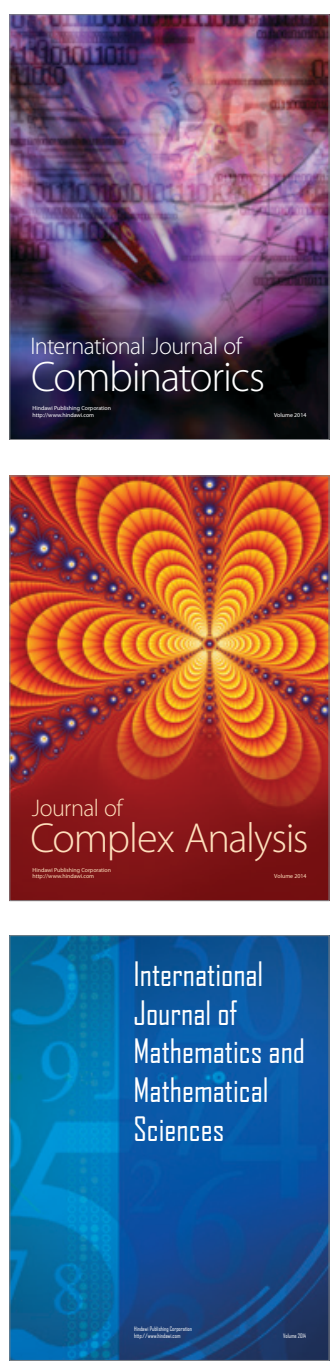
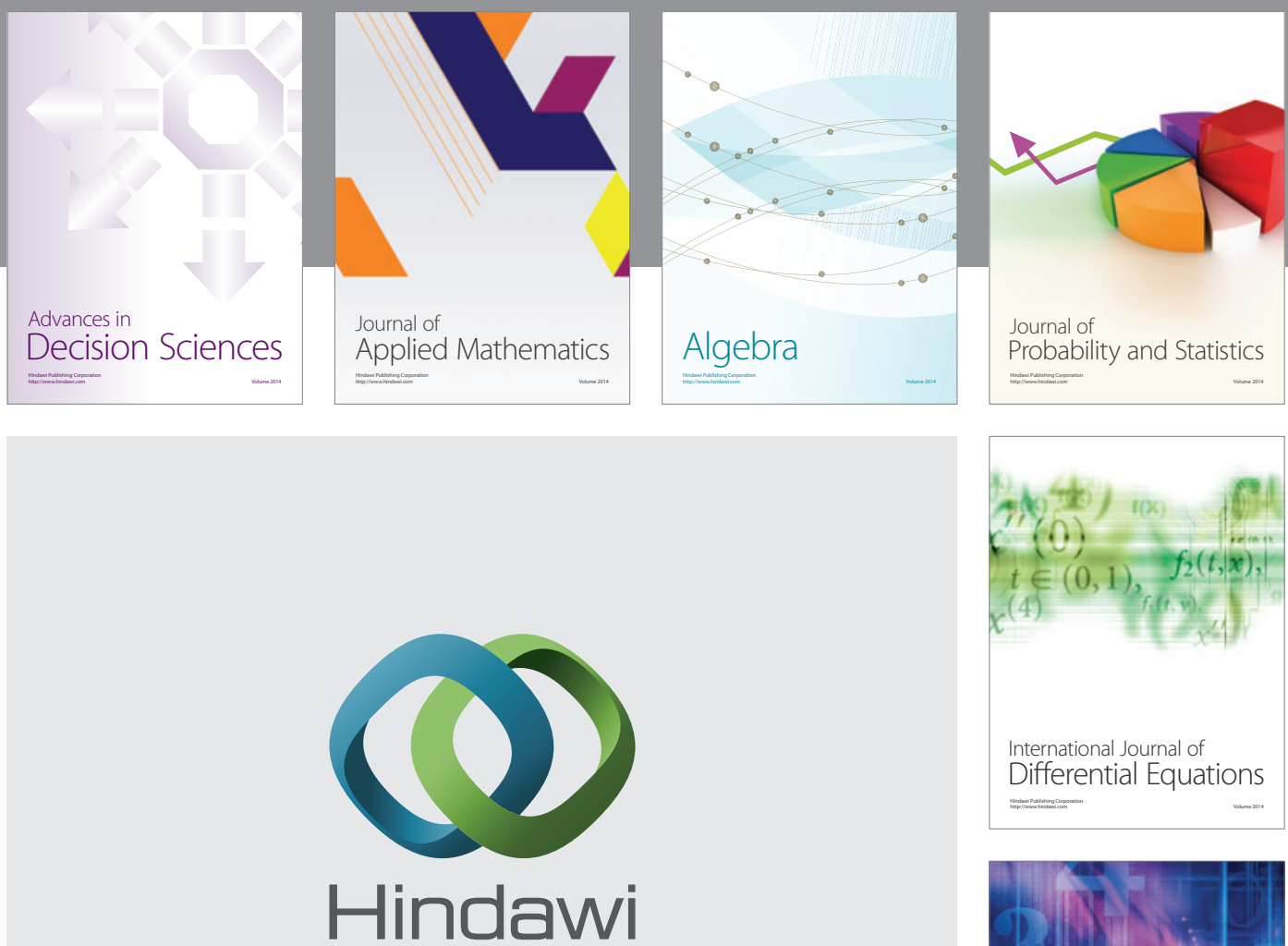

Submit your manuscripts at http://www.hindawi.com
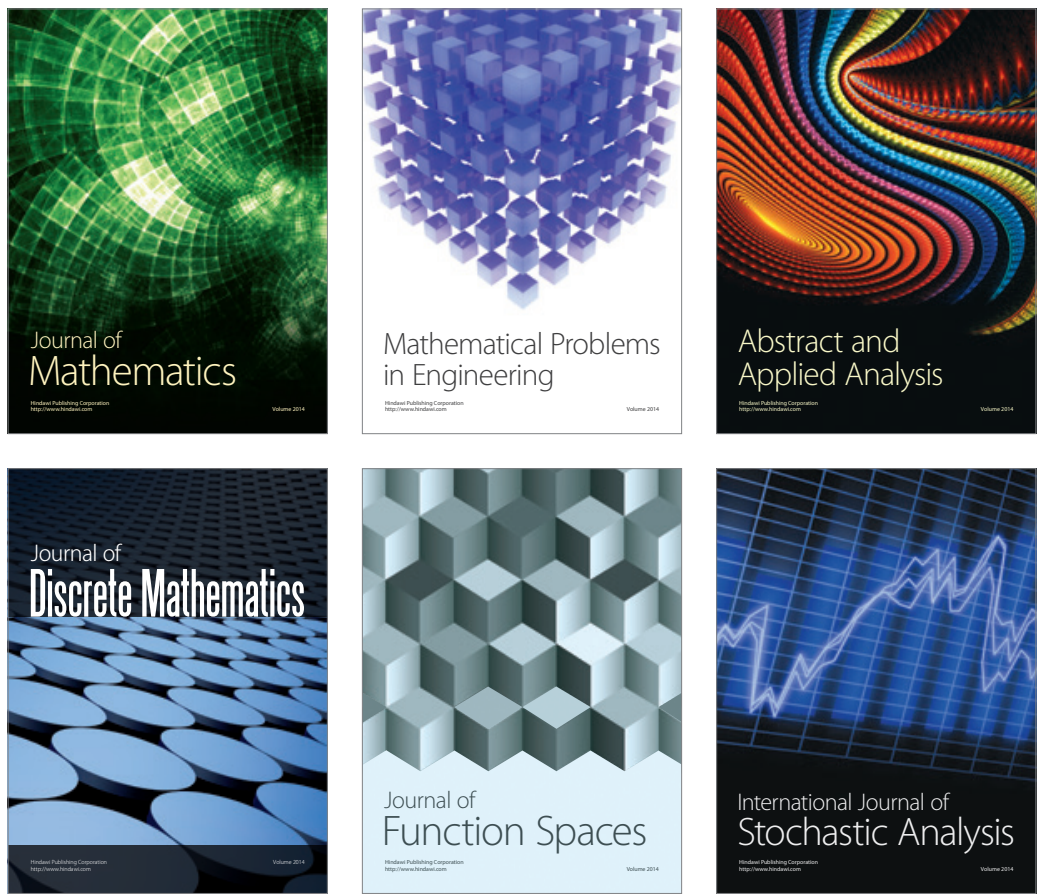

Journal of

Function Spaces

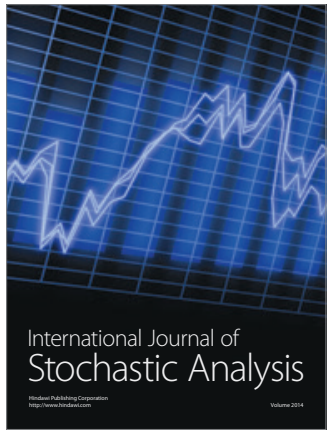

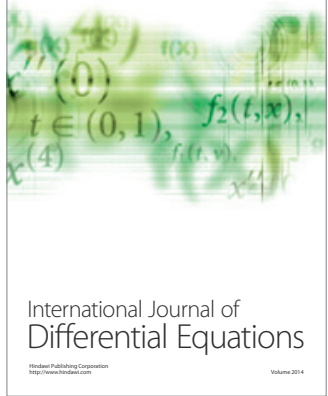
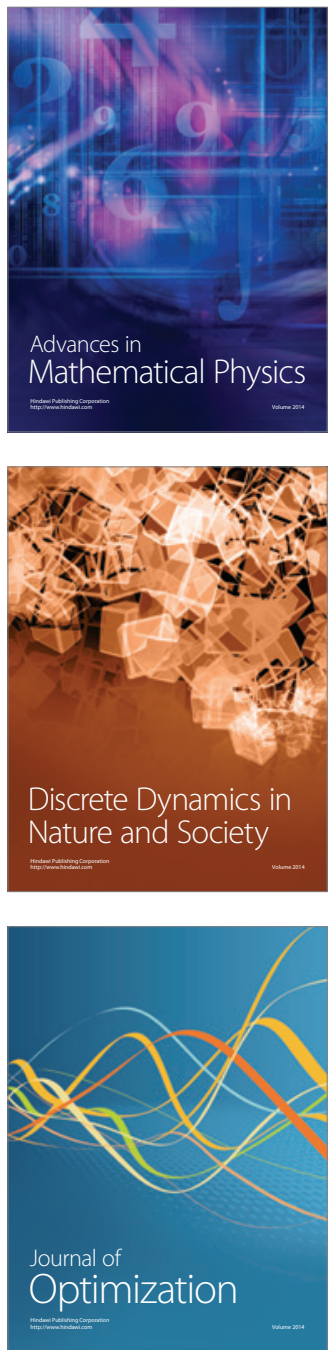